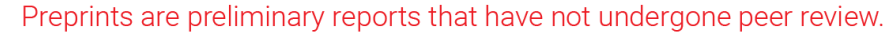 They should not be considered conclusive, used to inform clinical practice, or referenced by the media as validated information. \\ Crystal and Electronic Structure Engineering of Tin Monoxide by External Pressure
}

\section{Kun Li}

Northwestern Polytechnical University School of Materials Science and Engineering

Junjie Wang ( $\nabla$ wang.junjie@nwpu.edu.cn )

Northwestern Polytechnical University School of Materials Science and Engineering https://orcid.org/0000-0002-6428-2233

Vladislav A. Blatov

Samara State Technical University

\section{Yutong Gong}

Northwestern Polytechnical University School of Materials Science and Engineering

Naoto Umezawa

Samsumg Electronics

Tomofumi Tada

Tokyo Institute of Technology

Hideo Hosono

Tokyo Institute of Technology

Artem R. Oganov

Skolkovo Institute of Science and Technology

\section{Research Article}

Keywords: Tin monoxide, van der Waals, topological relationship, phase transition, band gap

Posted Date: December 17th, 2020

DOI: https://doi.org/10.21203/rs.3.rs-128647/v1

License: (c) (i) This work is licensed under a Creative Commons Attribution 4.0 International License.

Read Full License 


\title{
Crystal and Electronic Structure Engineering of Tin Monoxide by External Pressure
}

\author{
Kun Li ${ }^{a, b}$, Junjie Wang ${ }^{a, b},{ }^{*}$, Vladislav A. Blatov ${ }^{c, b, d}$, Yutong Gong ${ }^{a}$, Naoto \\ Umezawa $^{e}$, Tomofumi $\operatorname{Tada}^{f}$, Hideo Hosono $^{f}$, Artem R. Oganov $^{g}, a, b, h$
}

${ }^{a}$ State Key Laboratory of Solidification Processing, Northwestern Polytechnical University, Xi'an, Shaanxi 710072, People's Republic of China.

${ }^{b}$ International Center for Materials Discovery, School of Materials Science and Engineering, Northwestern Polytechnical University, Xi'an, Shaanxi 710072, People's Republic of China.

' Samara Center for Theoretical Materials Science (SCTMS), Samara State Technical University, Molodogvardeyskaya St. 244, Samara, 443100, Russia.

${ }^{d}$ Samara Center for Theoretical Materials Science (SCTMS), Samara University, Ac. Pavlov St. 1, Samara 443011, Russia.

${ }^{e}$ Semiconductor R\&D Center, Samsung Electronics, 1, Samsungjeonja-ro, Hwaseongsi, Gyeonggi-do 18448, South Korea.

${ }^{f}$ Materials Research Center for Element Strategy, Tokyo Institute of Technology, 4259 Nagatsuta-cho, Midori-ku, Yokohama, Kanagawa 226-8503, Japan.

${ }^{g}$ Skolkovo Institute of Science and Technology, 3 Nobel Street, Moscow 143026, Russia.

${ }^{h}$ Moscow Institute of Physics and Technology, 9 Institutskiy Lane, Dolgoprudny city, Moscow Region 141700, Russia.

\section{Corresponding Author}

*Email: wang.junjie@nwpu.edu.cn

\section{Abstract}


Although tin monoxide $(\mathrm{SnO})$ is an interesting compound due to its $p$-type conductivity, a widespread application of $\mathrm{SnO}$ has been limited by its narrow band gap of $0.7 \mathrm{eV}$. In this work, we theoretically investigate the structural and electronic properties of several $\mathrm{SnO}$ phases under high pressure through employing van der Waals $(\mathrm{vdW})$ functionals. Our calculations reveal that a metastable $\mathrm{SnO}(\beta-\mathrm{SnO})$, which possesses space group $P 2{ }_{1} / c$ and a wide band gap of $1.9 \mathrm{eV}$, is more stable than $\alpha-\mathrm{SnO}$ at pressures higher than $80 \mathrm{GPa}$. Moreover, a stable (space group $P 2 / c$ ) and a metastable (space group Pnma) phases of SnO appear at pressures higher than $120 \mathrm{GPa}$. Energy and topological analyses show that $P 2 / c-S n O$ has a high possibility to directly transform to $\beta-\mathrm{SnO}$ at around $120 \mathrm{GPa}$. Our work also reveals that $\beta-\mathrm{SnO}$ is a necessary intermediate state between high-pressure phase Pnma-SnO and low-pressure phase $\alpha$ $\mathrm{SnO}$ for the phase transition path Pnma-SnO $\rightarrow \beta-\mathrm{SnO} \rightarrow \alpha-\mathrm{SnO}$. Two phase transition analyses indicate that there is a high possibility to synthesize $\beta$-SnO under high-pressure conditions and have it remain stable under normal pressure. Finally, our study reveals that the conductive property of $\beta-\mathrm{SnO}$ can be engineered in a lowpressure range $(0-9 \mathrm{GPa})$ through a semiconductor-to-metal transition, while maintaining transparency in the visible light range.

\section{Keywords}

Tin monoxide, van der Waals, topological relationship, phase transition, band gap

\section{Introduction}

Tin monoxide $(\mathrm{SnO})$ of the space group $P 4 / \mathrm{nmm}$ is a $p$-type semiconductor with an indirect band gap $(0.7 \mathrm{eV})$ [1] and shows promise as anode material in lithium rechargeable battery [2] and active layer of thin film transistors [1]. The bipolarity of $\mathrm{SnO}$ was first reported by experiment [3] and recently validated with theoretical calculations [4]. However, the narrow band gap of $\mathrm{SnO}(\sim 0.7 \mathrm{eV})$ hinders its extensive application in various related fields, e.g. solar cell [5]. To overcome this shortcoming, some efforts have been made including as modifying the band gap of $\mathrm{SnO}$ and synthesizing a new $\mathrm{SnO}$ with a wider band gap. For instance, Peng et al. [5] proved that alloying with divalent cations $(\mathrm{Mg}, \mathrm{Ca}, \mathrm{Sr}$ and $\mathrm{Zn})$ could engineer the band structure of $\mathrm{SnO}$ to a suitable semiconductor for photovoltaics. Wang et al. [6] predicted a series of 
novel structures of mixed-valence tin oxides by combining density functional theory (DFT) calculations with the evolutionary algorithm USPEX (Universal Structure Predictor: Evolutionary Xtallography) [7-9]. Among these predicted structures, a novel phase of layered $\mathrm{SnO}$ with the space group $P 2{ }_{1} / c$ attracts us due to its excellent stability and interesting electronic properties at ambient pressure [6]. The new $\mathrm{SnO}$ phase is only $0.03 \mathrm{eV} /$ atom less stable than the most stable $\alpha-\mathrm{SnO}$ phase at $0 \mathrm{GPa}$ and is more stable than other previously reported $\mathrm{SnO}$ structures $[6,10]$. Furthermore, it was confirmed dynamically stable by phonon dispersion calculations and named as beta-phase $\mathrm{SnO}(\beta$ $\mathrm{SnO})$ [6]. Moreover, DFT calculations revealed that $\beta-\mathrm{SnO}$ has a band gap of around $1.7 \mathrm{eV}$, which is suitable for photo-functional applications [6]. However, no clear clue as to how such a new phase can be synthesized experimentally was provided. From perspective of crystallography, $\beta-\mathrm{SnO}$ can be regarded as a distortion of $\alpha-\mathrm{SnO}$ and its space group $P 2{ }_{1} / c$ is a subgroup of the space group $P 4 / \mathrm{nmm}$. Therefore, it may be expected that $\beta-\mathrm{SnO}$ can be obtained through a phase transition from $\alpha$-SnO under specific condition, e.g. high pressure. In recent years, high-pressure synthesis has attracted extensive interest due to its various advantages such as synthesizing new materials with seemingly impossible structures and compositions at ambient pressure, e.g. $\mathrm{NaCl}_{3}[11], \mathrm{KCl}_{3}[12]$ and $\mathrm{Na}_{2} \mathrm{He}$ [13]. In fact, high-pressure study of $\alpha$-SnO was performed experimentally once, but no high-pressure phase transition was observed in the conducting pressure range from 0 to $51 \mathrm{GPa}$ [14]. Here, a systematic theoretical investigation was carried out to explore the possibility of obtaining $\beta$-SnO under highpressure conditions up to $150 \mathrm{GPa}$. Previous study revealed that conventional local and semi-local density functionals in DFT cannot well deal with the structures of layered tin monoxides $[6,15]$, which are bound by van der Waals (vdW) forces. The vdW correction within DFT has been validated as a useful way to improve the calculation for layered structures. Govaerts et al. [15] introduced different vdW functionals to rebuild the crystal structures and the electronic properties of $\alpha-\mathrm{SnO}$ at $0 \mathrm{GPa}$, and observed that the optB86b functional [16] gave the closest lattice parameters to the experimental data. Allen et al. [4] proposed that the PBE0-vdW approach, with $15 \%$ Fock exchange, is also a good choice for calculation of both lattice parameters and electronic properties of $\alpha$-SnO. However, a detailed test of available vdW functionals was still absent especially for the application at high pressure, which is necessary to the high-pressure simulation performed in our work. 
In our study, we first tested all available vdW functionals to rebuild the crystal structures of $\alpha-\mathrm{SnO}$ from 0 to $50 \mathrm{GPa}$ using the result of available high-pressure experiment of $\alpha-\mathrm{SnO}[14]$ as reference. The tested $\mathrm{vdW}$ functionals include D2 and D3 corrections of Grimme [17], Tkatchenko-Scheffler method [18], self-consistent screening in Tkatchenko-Scheffler method (TS-SCS) [19], many-body dispersion [1920], SCAN-rVV10 functionals [21] and methods combined with hybrid functional HSE06 [22]. Among these tested functionals, HSE06-TS-SCS, a combination of HSE06 and TS-SCS, was suggested to be the best method under ambient pressure to calculate the crystal structure and electronic properties of $\alpha$-SnO with high accuracy, while DFT-D3-BJ [23] shows a competitiveness under the high pressure. Furthermore, the phase transitions of $\mathrm{SnO}$ under high-pressure conditions (0-150 GPa) were carefully studied through employing the DFT-D3-BJ functional. The calculation suggests that $\alpha$ $\mathrm{SnO}$ might transform to $\beta-\mathrm{SnO}$ at pressures higher than $80 \mathrm{GPa}$ and two new phases of SnO with space groups $P 2 / c$ and Pnma will appear at high pressures of 120-150 GPa. The possibility of synthesizing $P 2 / C-\mathrm{SnO}$ under high pressure and subsequently transforming to $\beta-\mathrm{SnO}$ upon the release of pressure is revealed. Moreover, energy, structure and topological analysis revealed that both $P 2 / c$ - and Pnma-SnO prefer to transfer to $\beta-\mathrm{SnO}$ when external pressure is released, suggesting that one could expect the formation of $\beta-\mathrm{SnO}$ via high-pressure synthesis from $\mathrm{Sn}$ and oxygen. The electronic properties of $\beta$-SnO under low external pressure $(0-20 \mathrm{GPa})$ were studied using the DFT-D3-BJ functional. It is revealed that in a range of relatively low external pressures, the band gap of $\beta-\mathrm{SnO}$ can be continuously adjusted from 1.9 to almost $0 \mathrm{eV}$. Finally, we found that the conductivity and optical transparency of $\beta$-SnO might be engineered by external pressure.

\section{Computation methods}

All DFT calculations in this study were conducted using the Vienna ab initio simulation package (VASP) [24]. Structure relaxation and electronic property calculation were performed through combining vdW correction and the PerdewBurke-Ernzerhof (PBE) [25] functionals. The projector augmented wave (PAW) [2627] potentials treating $5 s^{2} 5 p^{2} 4 d^{10}$ and $2 s^{2} 2 p^{4}$ as valence electrons were used for $\mathrm{Sn}$ and $\mathrm{O}$ atoms, respectively. A plane-wave kinetic energy cutoff of $900 \mathrm{eV}$ was set for all calculations. Monkhorst-Pack [28] Brillouin zone sampling grid with a resolution of $2 \pi \times 0.03 \AA^{-1}$ was used in structure relaxations, while a k-mesh with spacing of around 
$2 \pi \times 0.015 \AA^{-1}$ was applied in the electronic structure calculations. For the electronic structure calculation, our results were converged to within $10^{-4} \mathrm{eV} / \mathrm{atom}$. For the structure relaxations, the simulation will stop until the force is converged to less than $0.001 \mathrm{eV} / \AA$. Phonon dispersion calculations were carried out using the finite displacement method as implemented in the PHONOPY code [29]. The Hartree-Fock mixing parameter and the screening parameter $\mu$ were respectively set to $25 \%$ and 0.2 $\AA^{-1}$ for the screened hybrid functional given by Heyd, Scuseria, and Ernzerhof (HSE06) [22] adopted for structure relaxation and electronic structure calculation.

Standard PBE and HSE06 functionals do not give a good description of the layered $\mathrm{SnO}$ structures because the dispersion forces between layers cannot be properly accounted for. To overcome this problem, different corrections of DFT-D, vdW-DF, DFT-TS, DFT-D3-BJ and SCAN-rVV10 were tested [14, 18-19, 30-31]. DFT-D adds a correction term to a standard Kohn-Sham DFT result, which could be interpreted in the following form [23]:

$$
E_{d i s p}^{D F T-D}=-\frac{1}{2} \sum_{A \neq B} \sum_{n=6,8,10, \ldots} s_{n} \frac{C_{n}^{A B}}{R_{A B}^{n}} f_{d a m p}\left(R_{A B}\right) .
$$

Where the summations are over all atoms, $C_{n}^{A B}$ denotes the nth-order dispersion coefficient (orders $\mathrm{n}=6,8,10, \ldots$ ) between atoms $\mathrm{A}$ and $\mathrm{B}$, and $R_{A B}$ means length of atom pair $\mathrm{AB}$. The term $s_{n}$ can be used to adjust the correction to the repulsive behavior of the chosen exchange-correlation density functional. The normal DFT-D3 method adopts zero-dumping function $f_{\text {damp. }}$. As a result, $E_{\text {disp }}^{D F T-D}$ tends to be 0 while the $R_{A B}$ approaches 0. Notably, the methods labled "DFT-*" mean the combination of vdW functionals and the PBE functionals. Among these methods, the DFT-D3-BJ is built by introducing Becke-Jonson (BJ) damping to D3 method, leading to a constant contribution of $E_{\text {disp }}^{D F T-D}$ to total energy, which is more reasonable than zero-damping. In our work, DFT-D3-BJ correction shows a good performance of optimizing the structure of $\alpha-\mathrm{SnO}$ in the whole pressure range, and subsequently is used to study the structural and electronic properties of $\beta-\mathrm{SnO}$ under external pressure.

Combined with structural relaxation using VASP code, USPEX has been proved as a successful tool to predict novel structures [6, 11-13] and properties [27, 32-33] especially at high pressure. Considering the high pressure we applied in the present study, USPEX is an ideal tool to search the globally stable phase of SnO at high pressure. 
In this work, three fixed-composition searches at pressure of $150 \mathrm{GPa}$ were performed for $\mathrm{SnO}$ in unit cells of containing 8,12 and 16 atoms, respectively. The global optimization was carried out by USPEX to generate the potentially stable structures. All structures of the first generation were randomly produced and were optimized by DFT-D3-BJ using VASP to compare their enthalpies for the local optimization. $40 \%$ of the structures owing the lowest enthalpies in the first generation were used to generate next generation by variation operations including heredity (40\%), lattice mutation (20\%), transmutation (20\%) and random generator (20\%). The global and local optimizations are repeated alternately until the most stable structure was not changed for more than 8 or 16 generations.

Topological analysis for searching transition pathways among different $\mathrm{SnO}$ phases was performed using the program package ToposPro and the database of topological types (TTD collection) [34]. The RCSR three-letter symbols [35] and the symbols for derived nets [36] were used to designate the network topologies. The structures of all phases were represented as periodic nets and the transitions between different phases were considered as transformations between the nets. The network model of reconstructive phase transitions (NMRT) [37] was applied to find the transition topological structures (subnets and supernets) between the initial and final phases. A subnet or a supernet can be obtained from a given periodic net by breaking existing bonds or creating new bonds, respectively. According to NMRT, if crystal structures $\mathrm{A}$ and $\mathrm{B}$ have different topologies, i.e. the corresponding periodic nets are not homeomorphic, then $\mathrm{A}$ and $\mathrm{B}$ cannot be transformed to each other without breaking and/or creating bonds. If $\mathrm{A}$ is a supernet of $\mathrm{B}$, i.e. contains $\mathrm{B}$, or vice versa $\mathrm{A}$ is a subnet of $\mathrm{B}$, i.e. can be embedded into $\mathrm{B}$, the transition $\mathrm{A} \rightarrow \mathrm{B}$ is direct and can be respectively performed by breaking or creating some bonds in A. In other cases, for example, when the coordination of atoms is the same in $\mathrm{A}$ and $\mathrm{B}$, the transition passes through at least one intermediate net $\mathrm{C}$, which is a common subnet $(\mathrm{C} 1)$ or a common supernet $(\mathrm{C} 2)$ of $\mathrm{A}$ and $\mathrm{B}$. $\mathrm{C} 1$ consists of all bonds, which exist in both $\mathrm{A}$ and $\mathrm{B}$, while $\mathrm{C} 2$ includes all bonds from $\mathrm{A}$ and $\mathrm{B}$. Thus, to pass from $\mathrm{A}$ to $\mathrm{B}$ through $\mathrm{C} 1$, one should break those bonds in $\mathrm{A}$, which do not exist in $\mathrm{B}$, and then create new bonds, which exist in $\mathrm{B}$, but not in A. Vice versa, to perform the transition through C2, one should perform these operation in an opposite order. That is why $\mathrm{C} 1$ and $\mathrm{C} 2$ form a pair subnet/supernet, which characterizes the topological transformations during the transition. ToposPro 
enables one to find all common subnets and supernets of any nets and, hence, to reveal topological transformations of crystalline solids in an automated way.

The optical properties were obtained by combination of VASP and VASPKIT [38], while the absorption coefficient $I(\omega)$ was calculated by the following equation [39]:

$$
I(\omega)=\sqrt{2} \omega\left[\sqrt{\varepsilon_{1}(\omega)^{2}+\varepsilon_{2}(\omega)^{2}}-\varepsilon_{1}(\omega)\right]^{\frac{1}{2}}
$$

where $\varepsilon_{1}(\omega)$ and $\varepsilon_{2}(\omega)$ are the real and imaginary parts of the dielectric function, respectively. The VASPKIT were also used to calculate the wavefunction characters. In addition, the crystal orbital overlap populations (COOP) were examined by LOBSTER [40] to analyze the chemical bonds.

\section{Results and disscution}

\subsection{The comparison of vdW functionals}

To verify the feasibility of vdW functional to layered $\mathrm{SnO}$ system, we first optimized the structure of $\alpha-\mathrm{SnO}$ in the pressure range from 0 to $50 \mathrm{GPa}$ in comparison with the available experimental data [14] (Table S1). The calculated results from different functionals at $0,10,20,30,40,50 \mathrm{GPa}$ are shown in Fig. 1, Figures S1-5 and Tables S2-7. Fig. 1 shows that both PBE and HSE06 functionals overestimate the lattice parameter $c$ dramatically at $0 \mathrm{GPa}$, whereas lattice parameter $a$ can be well estimated, leading to a significant deviation of the $a / c$ ratio and unit cell volume from experimental values. This result proved again that the lattice parameter $c$ is drastically influenced by the interlayer vdW force. In addition, the revPBE and rPW86 functionals [41-42] are not suitable for relaxing $\alpha-\mathrm{SnO}$ that the lattice parameters obtained from these two methods show huge error at each pressure.

As expected, the introduction of $\mathrm{vdW}$ corrections can greatly improve the calculation accuracy of lattice parameter $c$ of $\alpha-\mathrm{SnO}$. The DFT-D2 and DFT-D3 functionals developed by Grimme et al. $[17,31]$ give $c$ values close to the experimental data while slightly overestimating the lattice parameter $a$. However, the DFT-TS functional developed by Tkatchenko et al. [18] underestimates the lattice parameter $c$ notably. Similar phenomena are observed in the calculations using MBD-202 and MBD-212 functionals, which considered many-body dispersion interactions among 
atoms [19]. The constant $a$ calculated by the SCAN-rVV10 functionals developed by Peng et al. [21] shows a small error, but the lattice constant $c$ is seriously underestimated. On the contrary, the DFT-TS-SCS functional developed by Tkatchenko et al. [19] can perfectly avoid the underestimation of the lattice parameter $c$, while a significant overestimation of the parameter $a$ is observed. By combining the hybrid functional HSE06 and the vdW functional DFT-TS-SCS, the HSE06-TS-SCS functional can provide the best estimation of lattice parameters $a, c, a / c$ and volume for $\alpha-\mathrm{SnO}$ at 0 GPa (Fig. 1). The estimated lattice parameters $a$ and $c$ at 0 GPa only slightly deviate from experimental values with errors of $0.08 \%$ and $-0.62 \%$, respectively. However, the lattice parameters yielded by HSE06-MBD-212 and HSE06-TS are worse than those obtained from HSE06-TS-SCS. It is noteworthy that the obtained structural parameters with the optB86b-vdW are the second best to HSE06-TS-SCS, which is in line with the previous studies [15].

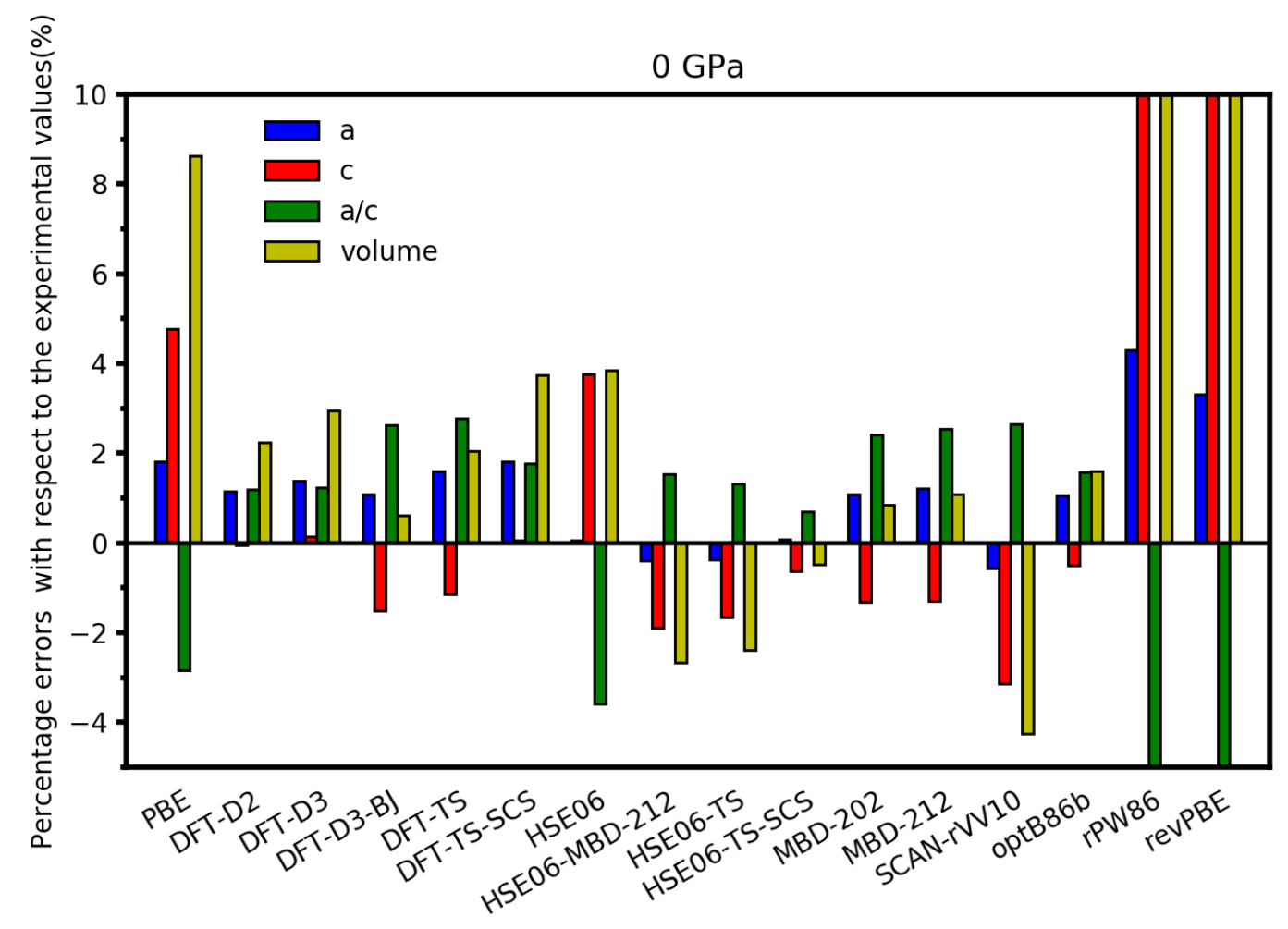

Fig. 1. Percentage errors of calculated $a$ and $c, a / c$ ratio and volume of the unit cell of $\alpha$-SnO using different functionals with respect to the experimental values at $0 \mathrm{GPa}$. 
Although the deviation of calculated parameters from experimental values increases with the increase of external pressure (Figures S1-5), the HSE06-TS-SCS, DFT-D3-BJ, MBD-202, MBD-212 and optB86b functionals still exhibit their competitiveness, considering the good coherence between calculated lattice parameters and experimental values. In principle, the HSE06-TS-SCS functional is the first choice to deal with Sn-O layered structures. However, in consideration of balancing calculation accuracy and cost under high pressure, we alternatively used the DFT-D3$\mathrm{BJ}$ functional in the following calculations.

\subsection{The possibility of high-pressure synthesis}

To compare the stabilities of $\alpha$ - and $\beta$-SnO under pressure, the two structures were fully relaxed at pressures from 0 to $150 \mathrm{GPa}$ employing vdW functional DFT-D3$\mathrm{BJ}$ (Fig. 3a). We found that the enthalpy of $\beta-\mathrm{SnO}$ becomes lower than that of $\alpha-\mathrm{SnO}$ at pressures higher than $80 \mathrm{GPa}$, indicating that $\beta-\mathrm{SnO}$ is more stable than $\alpha-\mathrm{SnO}$ at pressures higher than $80 \mathrm{GPa}$. Furthermore, to obtain the most stable $\mathrm{SnO}$ phase at high pressures, crystal structure searches for $\mathrm{SnO}$ were carried out using USPEX at $150 \mathrm{GPa}$. Two new phases of $\mathrm{SnO}$ with space groups $P 2 / c$ and Pnma were identified. At $150 \mathrm{GPa}$, the calculated enthalpies of $P 2 / c$ - and Pnma-SnO are 0.124 and $0.012 \mathrm{eV} /$ atom lower than that of $\alpha-\mathrm{SnO}$, respectively (Fig. 3a).

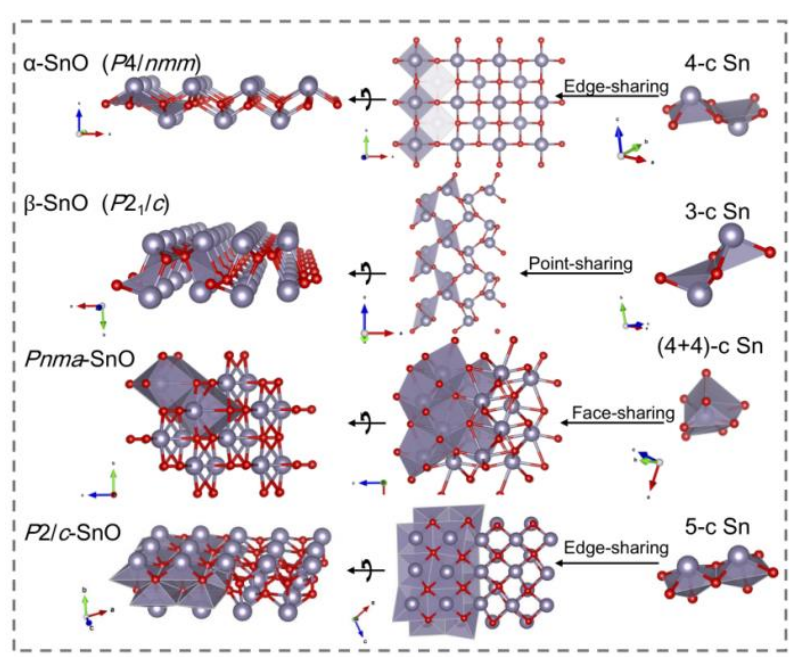

Fig. 2 Crystal structures of $\alpha-\mathrm{SnO}, \beta-\mathrm{SnO}, P n m a-\mathrm{SnO}$ and $P 2 / c-\mathrm{SnO}$ 
The phonon calculations show that $P 2 / c$-SnO is dynamically stable in the pressure range of 120-150 GPa (Fig. S6). The imaginary frequencies were observed in the phonon calculations at $0-110 \mathrm{GPa}$ for $P 2 / c-\mathrm{SnO}$ (Fig. S6), which suggests this phase will not be stable under ambient condition. Furthermore, the calculated enthalpy of $P 2 / c-\mathrm{SnO}$ is only $0.007 \mathrm{eV} /$ atom lower than that of $\beta-\mathrm{SnO}\left(P 2{ }_{1} / c\right)$ at $120 \mathrm{GPa}$ (where $P 2 / c-\mathrm{SnO}$ starts to be dynamically stable), while enthalpy of $\alpha-\mathrm{SnO}$ is $0.073 \mathrm{eV} /$ atom higher than that of $P 2 / c-\mathrm{SnO}$ (Fig. 3a). Fig. $3 \mathrm{~b}$ shows that the local structure of $P 2 / c$ $\mathrm{SnO}$ could be viewed as a slight deformation of that of $\beta-\mathrm{SnO}\left(P 2_{1} / c\right)$. The topological analysis of the $\mathrm{SnO}$ crystal structures using ToposPro [34] indicates that these two structures belong to the same topology at high pressure. In the $P 2 / c-\mathrm{SnO}$ phase all atoms are 5-coordinated, thus two more $\mathrm{Sn}-\mathrm{O}$ bonds are formed in $\beta-\mathrm{SnO}$ during the transition (Fig. 3c). The topology of the layers in the $P 2 / c-\mathrm{SnO}$ phase is known as $4^{4} \mathrm{Ia}$ 2-periodic sphere packing [43], which has the maximum $P 4 / \mathrm{mmm}$ space-group symmetry. Both $P 2 / c$ and $P 2_{1} / c$ are general subgroups of $P 4 / \mathrm{mmm}$, and the changes in symmetry are caused by a shift of the layers. Thus the $\beta-\mathrm{SnO} \rightarrow P 2 / c-\mathrm{SnO}$ or reversed $P 2 / c-\mathrm{SnO} \rightarrow \beta-\mathrm{SnO}$ transformations can be described as the topological reconstruction of each layer (Fig. 3c) followed by an appropriate shift of the layers. These results indicate the energy barrier of the phase transition at around $120 \mathrm{GPa}$ from $P 2 / c$-SnO to $\beta-\mathrm{SnO}\left(P 2_{1} / c\right)$ is much smaller than that to $\alpha-\mathrm{SnO}$. Therefore, it is highly expected that $P 2 / c$-SnO phase can be synthesized at high pressure condition and may transform to $\beta$ $\mathrm{SnO}\left(P 2_{1} / c\right)$ upon the release of external pressure at around $120 \mathrm{GPa}$.

Calculated phonon band structure shows that Pnma-SnO can be dynamically stable in the pressure range from 20 to $150 \mathrm{GPa}$ (Fig. S6). The calculated enthalpy of Pnma-SnO is lower than that of $\alpha-\mathrm{SnO}$ in the pressure range of $140-150 \mathrm{GPa}$ but is always higher than that of $P 2 / c-\mathrm{SnO}$, which suggests that Pnma-SnO is only a metastable phase in the studied pressure range. However, since the formation of compounds is often the interplay between thermodynamics and kinetics, the metastable Pnma-SnO may be synthesized by certain non-equilibrium experimental strategies. Therefore, the pressure-induced phase transition of Pnma-SnO was also hypothetically analyzed by topological method in this work.

It was revealed that the topological structures of $\alpha$ - and $\beta$-SnO belong to $\mathbf{s q l}$ and fes topologies [36], respectively, while the topology of Pnma-SnO is sra. Schematic 
diagrams of sql, fes and sra topologies (Fig. S7) and the details of topological analysis can be found in the Supporting Information. It is known that in the transition state the subnet (low-coordinated structure) coexists with the corresponding supernet (highcoordinated structure) because the stages of breaking and creating bonds can be reversed [37]. The corresponding supernet for the Pnma-SnO $\rightarrow \alpha-\mathrm{SnO}$ transition has the nia-5,5-P2 ${ }_{1} / c$ (derived from the NiAs type[44]) topology. This supernet contains all the $\mathrm{SnO}$ phases under consideration as its subnets; they can be obtained by breaking particular bonds in the supernet thus elucidating the reconstructive phase transitions (Fig. 3d). Therefore, the following transition sequences can be proposed at decreasing pressure: Pnma-SnO (Pnma; sra) $\rightarrow \beta-\operatorname{SnO}\left(P 2{ }_{1} / c\right.$; fes $) \rightarrow \alpha-\operatorname{SnO}(P 4 / n m m ;$ sql $)$, i.e. the symmetry of the initial four-coordinated Pnma-SnO decreases down to $P 2{ }_{1} / c$, then some $\mathrm{Sn}-\mathrm{O}$ bonds are broken yielding three-coordinated $\beta-\mathrm{SnO}$, then some new $\mathrm{Sn}-\mathrm{O}$ bonds are created giving rise to the four-coordinated alpha phase (Fig. 3d). This transition reflects the easiest geometrical transformation: indeed, the bond that is broken in the nia-5,5-P2 $1 / c$ transition state corresponds to one of the four weak bonds of the high-pressure Pnma-SnO.

Based on the above structure, topological and enthalpy analysis, one can conclude that $\beta-, P 2 / c$ - and $P n m a-S n O$ can be formed under high pressures and these new phases are likely to change or keep to $\beta-\mathrm{SnO}$ when the external pressure is removed. In addition, we believe the $\beta-\mathrm{SnO}$ will not transform to the $\alpha-\mathrm{SnO}$ without external effects due to the dynamical stability of both phase and the small difference in enthalpy of formation between $\beta-\mathrm{SnO}$ and $\alpha-\mathrm{SnO}$ at $0 \mathrm{GPa}$. Consequently, it is highly possible to synthesize $\beta-\mathrm{SnO}$ at high pressures and to keep it under ambient conditions.

In this section, we predicted a possible way for $\beta$-SnO synthesis, pressurization. The sequence of the phase transition of two stable high-pressure phases at $150 \mathrm{GPa}$ to $\beta-\mathrm{SnO}$ and $\alpha-\mathrm{SnO}$ are studied. The study shows the two different phases will preferentially transform to $\beta-\mathrm{SnO}$ when the pressure is released to $0 \mathrm{GPa}$. Therefore, it is suggested that the pressurization is a possible way for synthesis of $\beta$-SnO. Our work is a bold prediction of what may happen in the experiment based on the first-principles calculation and topological analysis. However, this work does not exclude the possibility of an unknown and stable structure under other pressures, which is complex and beyond this work. 


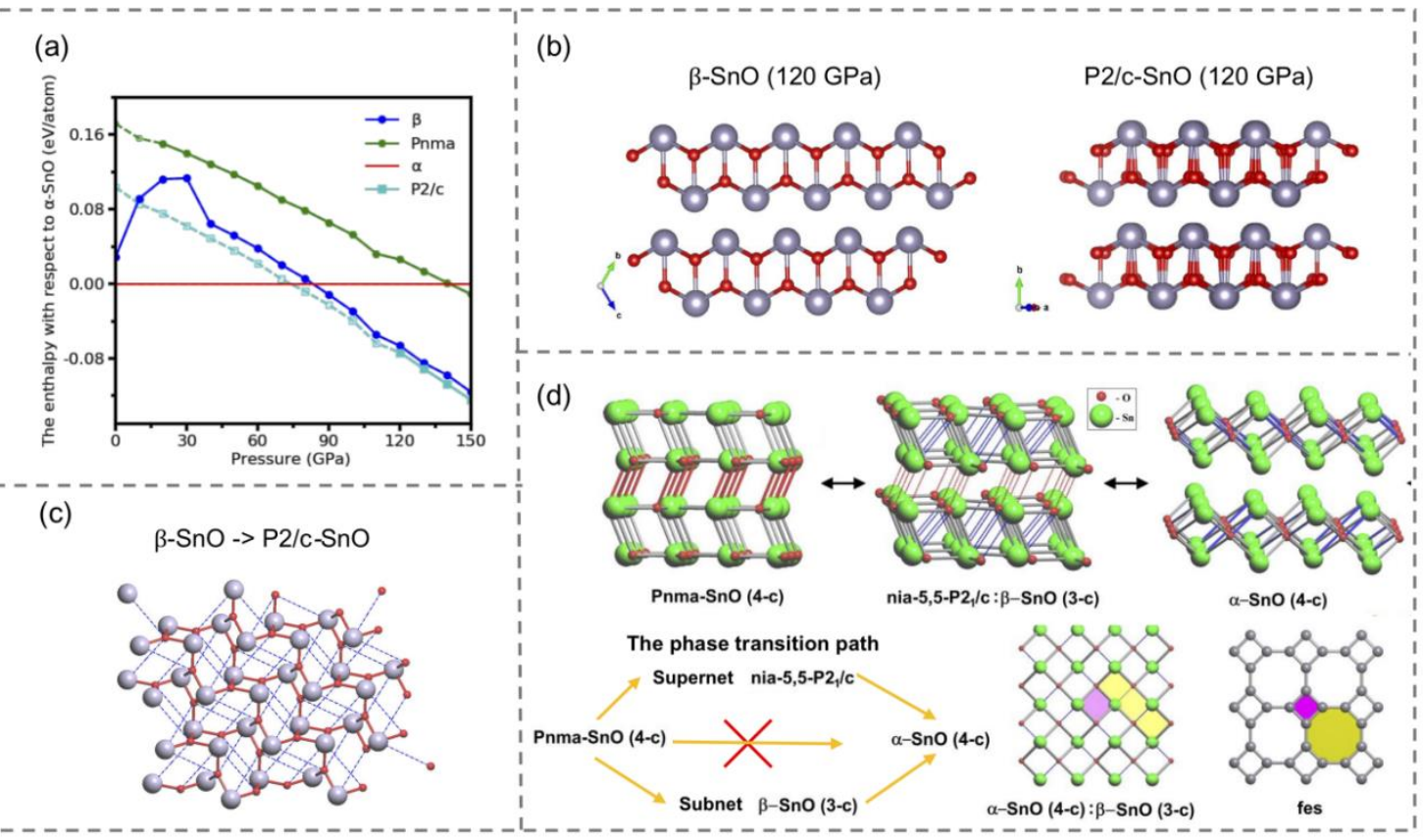

Fig. 3 (a) The enthalpy per atom for predicted tin monoxides as a function of external pressure with respect to $\alpha$-SnO. For Pnma- and P2/c-SnO, the dashed lines and hollow symbols indicate the dynamical instability at certain pressure. (b) A schematic diagram to demonstrate the structural similarity between $P 2 / c$-SnO and $\beta$-SnO $\left(P 2_{1} / c\right)$. (c) Transformation of $\beta$-SnO $\left(P 2_{1} / c\right)$ to $P 2 / c$ $\mathrm{SnO}$ by forming new bonds shown by thin blue lines. (d) The phase transition from high-pressure Pnma-SnO to $\alpha$-SnO through a supernet nia-5,5-P2 ${ }_{1} / c$ or $\beta$-SnO. Breaking the blue or red bonds in the supernet (upper middle of d) leads to $\alpha$-SnO or Pnma-SnO, respectively, while breaking both types of bonds gives rise to $\beta$-SnO; Relation between Pnma-SnO and $\beta$-SnO layers: breaking thin red bonds in Pnma-SnO leads to the $\beta$-SnO fes topology (upper left of d). The idealized fes net consisting of four- and eight-membered rings highlighted by magenta and yellow (bottom right of d), respectively. These rings correspond to the rings of the same color in the bottom middle picture.

\subsection{The electronic properties under external pressure}

The previous phase transition analysis shows that external pressure can manipulate the structures of $\mathrm{SnO}$ in a wide range. To explore the application prospect of $\beta$-SnO, we investigated the influence of pressure on the electronic structure of $\beta-\mathrm{SnO}$ in the following section. At $0 \mathrm{GPa}$, the band structure calculated by HSE06-TS-SCS shows a direct band gap of $1.9 \mathrm{eV}$ (Fig. S8). Due to the huge computational cost using HSE06TS-SCS, the vdW functional DFT-D3-BJ is recommended in high-pressure study for its excellent estimation for lattice parameters of $\alpha-\mathrm{SnO}$ in a wide pressure range. Indeed, the performance of DFT-D3-BJ functional on the electronic structure 
calculation of $\beta-\mathrm{SnO}$ is fairly good that it gives a band gap of $1.21 \mathrm{eV}$ at $0 \mathrm{GPa}$, which is marginally narrower than that obtained by HSE06-TS-SCS. Although the PBE functional based methods may underestimate the band gap, the pressure-induced trend of electronic properties obtained by PBE functional based methods should be the same as that obtained by HSE06-TS-SCS. Therefore, the structures and electronic structures of $\beta$-SnO in the pressure range 0-20 GPa were calculated using DFT-D3-BJ functional. The lattice parameters for the relaxed $\beta-\mathrm{SnO}$ are listed in Table S8.

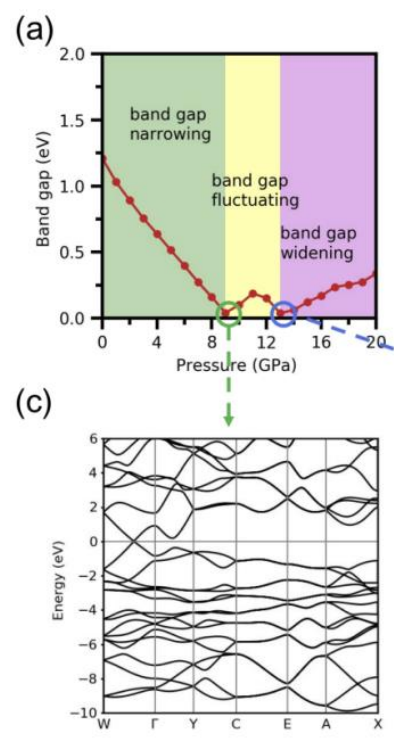

(b)

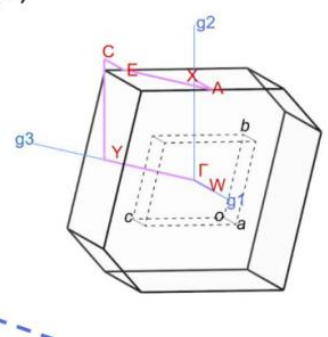

(e)
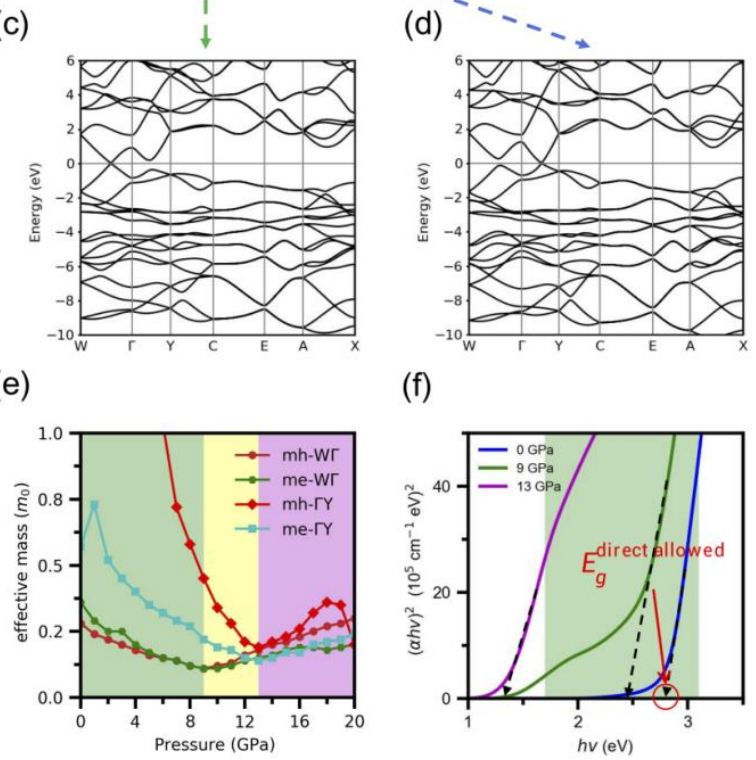

(f)

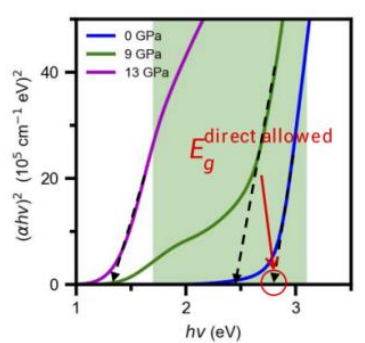

Fig. 4. (a) The variation of calculated band gap of $\beta-S n O$ using DFT-D3-BJ functional as a function of external pressure in the range of 0-20 GPa. (b) The Brillouin zone of $\beta$-SnO. The calculated band structures at pressures of (c) $9 \mathrm{GPa}$ and (d) $13 \mathrm{GPa}$. (e) Calculated effective mass of $\beta$-SnO along different high-symmetry directions; $m_{\mathrm{h}}$ and $m_{\mathrm{e}}$ represent the effective mass of hole and electron, respectively. (f) Calculated absorption spectra, $(\alpha h v)^{2}$ vs $h v$, for $\beta-\mathrm{SnO}$ at different pressures, where $\alpha$ and $h v$ are the absorption coefficient and photon energy, respectively. The direct allowed band gap has been measured by extrapolating straight portion of the curves $(\alpha h v)^{2}$ vs $h v$ [45]. Notably, the edge of the absorption spectrum at different pressures were shifted $+0.69 \mathrm{eV}$ to fit the band gap estimated by the HSE06-TS-SCS. The green area represents the energy range of visible light. 
The variation of calculated band gap of $\beta-\mathrm{SnO}$ as a function of external pressure is shown in Fig. 4a. In the pressure range of 0-9 GPa, a linear reduction of the band gap with the increase of pressure can be observed and the band gap becomes almost zero at $9 \mathrm{GPa}$. With the further increase of external pressure, the band gap of $\beta-\mathrm{SnO}$ gets wider and then closes again at around $13 \mathrm{GPa}$ (Fig. 4a). The calculated band structures of $\beta$ SnO along the k-path illustrated in Fig. $4 \mathrm{~b}$ at $9 \mathrm{GPa}$ and $13 \mathrm{GPa}$ are shown in Figs. 4c and $4 \mathrm{~d}$ for a detailed study of this unusual band gap closing. Two band crossings near Fermi level can be observed in those calculated band structures, which indicates a semiconductor-to-metal transition of $\beta$-SnO under external pressure. In addition, it shows that the CBM (conduction band minimum) and VBM (valence band maximum) of $\beta-\mathrm{SnO}$ are shifted from the $\mathrm{K}$ line $\mathrm{W}-\Gamma$ to $\Gamma-\mathrm{Y}$ with the increase of pressure from 9 GPa to $13 \mathrm{GPa}$. Then, the band gap of $\beta$-SnO gets open again (Fig. 4a) at pressures beyond $13 \mathrm{GPa}$. Finally, $\beta$-SnO becomes metallic at pressures above $50 \mathrm{GPa}$ (Fig. S9). This pressure-induced variation of band gap indicates that the $\beta$-SnO might be used as a pressure sensor [46].

The analysis of hole and electron effective mass demonstrates that pressure may alter the conductive property of $\beta$-SnO (Fig. 4e). It shows that all calculated effective mass along high-symmetry directions increase first and then decrease upon the increase of the external pressure. For instance, the $m_{\mathrm{h}}-\mathrm{W} \Gamma$ (hole effective mass along the $\mathrm{W}-\Gamma$ direction) can be as small as $0.12 m_{0}$, at $9 \mathrm{GPa}$. Moreover, the direct allowed band gap of the $\beta-\mathrm{SnO}$ is estimated to be around $2.8 \mathrm{eV}$ at $0 \mathrm{GPa}$, which is similar to that of $\alpha$ $\mathrm{SnO}(2.7 \mathrm{eV})[3]$ and $t-\mathrm{ZrOS}(2.5 \mathrm{eV})[47]$, implying the $\beta-\mathrm{SnO}$ could be a transparent semiconductor (Fig. 4f). A pressure-induced reduction of direct allowed band gap of the $\beta$-SnO can be also observed (Fig. 4f). However, a significant direct allowed band gap $(\sim 2.4 \mathrm{eV})$ can be observed at $9 \mathrm{GPa}$. At the pressures higher than $13 \mathrm{GPa}$, the direct allowed band gap is smaller than the $1.7 \mathrm{eV}$, the lower boundary of the visible light area. Combined with previous analysis of hole and electron effective mass, we believe high conductivity and transparency could be obtained in the $\beta-\mathrm{SnO}$ at suitable pressure, such as $9 \mathrm{GPa}$. 
(a)

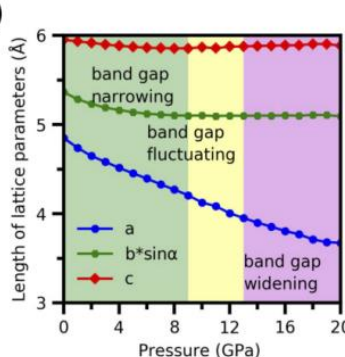

(c)

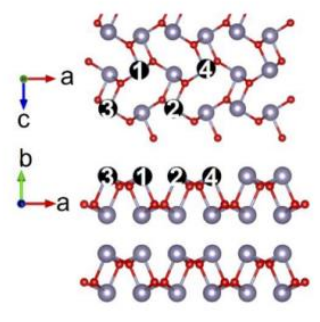

(b)

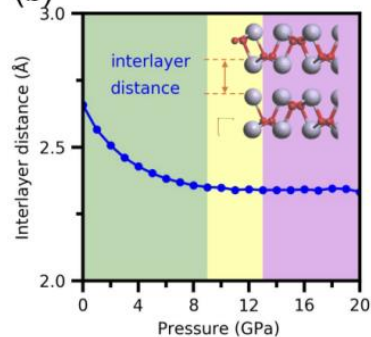

(d)

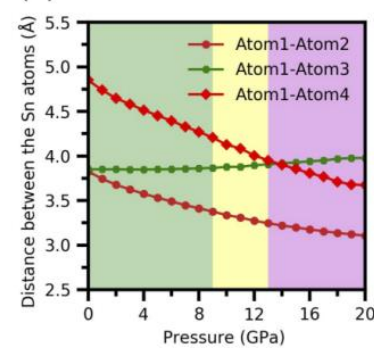

Fig. 5. The variation of (a) lattice parameters, (b) interlayer distance and (c, d) distance between different Sn atoms on the same surface as a function of external pressure in the range of 0-20 GPa.

It has been proved that the pressure-induced structural change can modulate electronic properties of compounds [48-49]. Therefore, the variation of lattice constants ( $a, b$ and $c$ ), distances of Sn-Sn pairs and interlayer distance $d$ in the pressure range 0$20 \mathrm{GPa}$ were calculated by DFT-D3-BJ (Fig. 5) and used to explain the band gap change of $\beta$-SnO. As crystal axis $b$ is not perpendicular to the plane of $a c, b * \sin \alpha$ instead of $b$ is shown in Fig. 5a ( $\alpha$ is the angle between axis $b$ and axis $c$ ). The distance between the Sn atoms of different layers was represented by the interlayer distance $d$ (Fig. 5b). The distances of atom1-atom2, atom1-atom3 and atom1-atom4 (Figs. 5c and 5d) respectively give the first, the second and the third nearest neighbor distances between Sn atoms on the same surface of the layer.

One can see that parameter $a$ (Fig. 5a) and the distances of atom1-atom2 and atom1-atom4 (Figs. 5c and 5d) decrease monotonically over the entire pressure range, while $c, b^{*} \sin \alpha$ (Fig. 5a), interlayer distance $d$ (Fig. 5b) and the distance of atom1atom 2 (Figs. $5 \mathrm{c}$ and $5 \mathrm{~d}$ ) show compression in the pressure range of band gap narrowing and then keep almost unchanged in the range of band gap fluctuating. In addition, the distance of atom1-atom 3 show almost no compression under pressure. When the $b{ }^{*} \sin \alpha$ and $d$ becomes less compressible, the band gap starts to fluctuate. Therefore, it seems the compressions of parameter $b^{*} \sin \alpha$ (Fig. 5a) and interlayer distance $d$ (Fig. 5b), which could affect the interlayer $\mathrm{Sn}$-Sn interaction, have the strongest influence on the band gap change of $\beta$-SnO in the pressure range of band gap narrowing and fluctuating. 
Moreover, the opening of the band gap at the pressure that is higher than $13 \mathrm{GPa}$ with the increase of parameter $c$ (Fig. 5a) and the distance of atom1-atom3 (Fig. 5d) indicates that the in-layer interactions influence the band gap opening at high pressure.

In order to gain insight into the relationship between the lattice deformation and band gap change of $\beta-\mathrm{SnO}$, the decomposed band structure and crystal orbital overlap populations (COOP) were examined and are shown in Fig. 6 and Fig. S10. One can see that $\mathrm{O}\left(p_{z}\right), \operatorname{Sn}(s)$ and $\operatorname{Sn}(p)$ contributes more to the band structure near the Fermi level than other orbits (Fig. S10). Based on these calculation results, band diagrams were constructed and are shown in Fig. 6. It shows that the VBM and CBM of $\beta-\mathrm{SnO}$ are determined by both the interlayer $\mathrm{Sn}-\mathrm{Sn}$ and intralayer $\mathrm{Sn}-\mathrm{O}$ interactions. The vertical interactions $\operatorname{Sn}\left(s+p_{z}\right)-\operatorname{Sn}\left(s+p_{z}\right)$ and $\operatorname{Sn}\left(p_{x}+p_{y}\right)-\operatorname{Sn}\left(p_{x}+p_{y}\right)$ across the $\mathrm{SnO}$ layer consist of the antibonding states of VBM and bonding states of CBM, as shown in Fig. 6. According to the molecular orbital theory, atomic $s$ orbital could bonds with $p_{x}, p_{y}$ and $p_{z}$, so $\operatorname{Sn}\left(s+p_{z}\right)-\operatorname{Sn}\left(s+p_{z}\right)$ is reasonable and suitable to reflect the interlayer chemical bonds. As the atomic $p_{x}, p_{y}$ and $p_{z}$ orbitals couldn't bonds with each other, the $\operatorname{Sn}\left(p_{x}+p_{y}\right)$ $\operatorname{Sn}\left(p_{x}+p_{y}\right)$ actually means $\left[\operatorname{Sn}\left(p_{x}\right)-\operatorname{Sn}\left(p_{x}\right)\right]+\left[\operatorname{Sn}\left(p_{y}\right)-\operatorname{Sn}\left(p_{y}\right)\right]$, which is used to exhibit the intralayer chemical bonds.

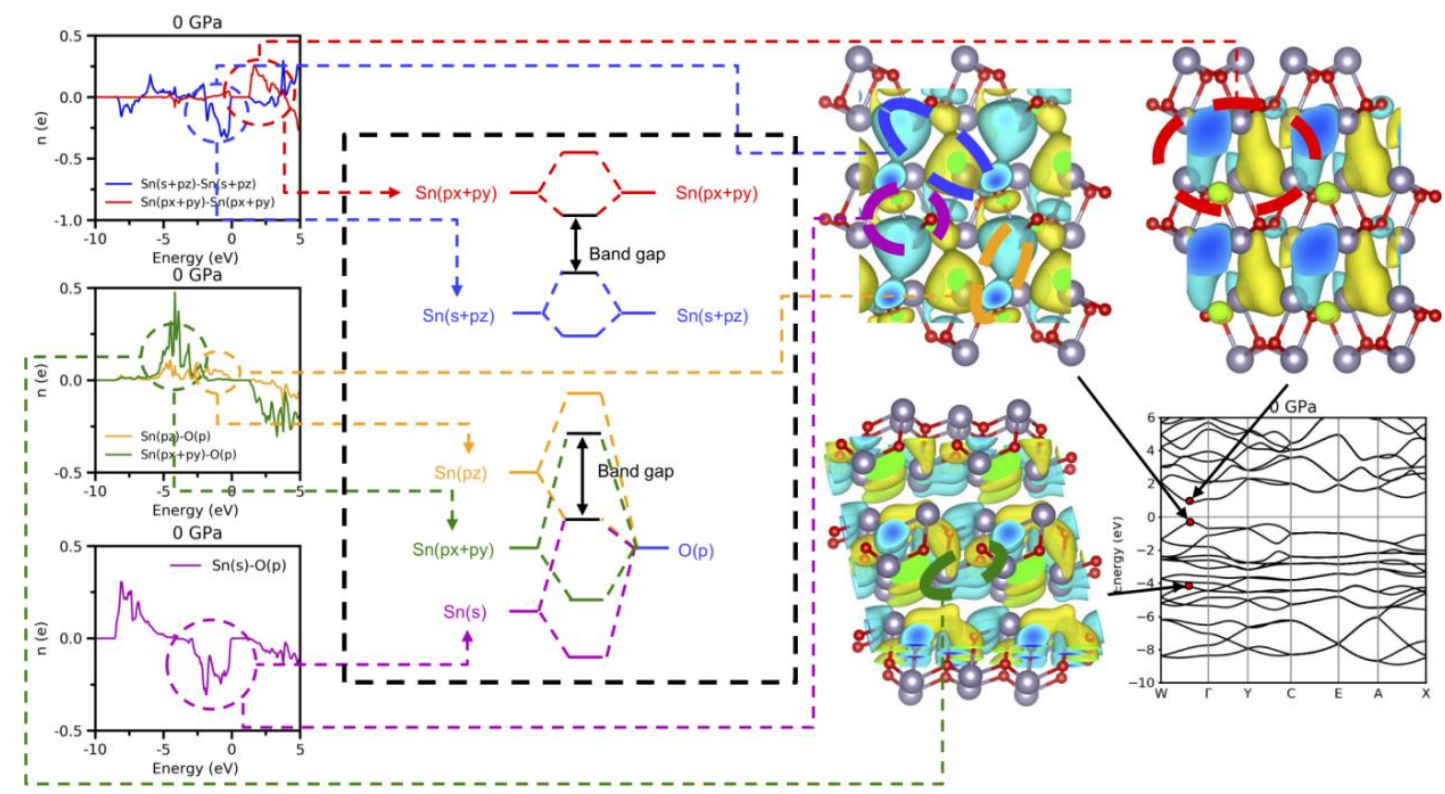

Fig. 6 The calculated crystal orbital overlap populations (COOP), schematic diagram of orbital interactions and the wave functions at representative points of the band structure for $\beta-\mathrm{SnO}$ at 0 
GPa. In this work, axis $z$ is perpendicular to the surface of SnO layer, while $x$ and $y$ are parallel to the axes $a$ and $c$, respectively.

With the increase of external pressure in the range of band gap closing, Fig. 5 shows that the variation of geometric parameters $b^{*} \sin \alpha$ and interlayer distance $d$ can directly influence the interlayer $\mathrm{Sn}-\mathrm{Sn}$ distance and interaction. The continuous decrease of $b^{*} \sin \alpha$ and interlayer distance $d$ is an indicator of the decreasing of $\mathrm{Sn}-\mathrm{Sn}$ interlayer distance, therefore, the Sn-Sn interaction will be enhanced gradually (Fig. 5). The corresponding splitting in the energy between the bonding and antibonding levels of $\operatorname{Sn}\left(s+p_{z}\right)-\operatorname{Sn}\left(s+p_{z}\right)$ and $\operatorname{Sn}\left(p_{x}+p_{y}\right)-\operatorname{Sn}\left(p_{x}+p_{y}\right)$ interactions get enhanced, which leads to the narrowing of the band gap. Therefore, the decease of the interlayer distance and enhanced interlayer $\mathrm{Sn}-\mathrm{Sn}$ interaction are responsible for the narrowing of band gap in the pressure range of 0-9 GPa. However, the oscillation and reopening (Fig. 4a) of the band gap with further increase in pressure cannot be explained by this mechanism.

As shown in Fig. S11, electron localization can be observed in the interlayer region, which corresponds to the $\operatorname{Sn}\left(s+p_{z}\right)$ states. With the increase of external pressure, it is expected that the pressure-induced decrease of interlayer distance of $\beta-\mathrm{SnO}$ will break the hybridization of $\operatorname{Sn}(s)$ and $\operatorname{Sn}\left(p_{\mathrm{z}}\right)$ orbitals and force part electrons into the layer. The calculated decomposed band structures (Fig. S12) reveals the charge transfer from $s$ to $p_{y}$ orbital of $\mathrm{Sn}$. Therefore, the intralayer $\operatorname{Sn}\left(p_{x}+p_{y}\right)-\mathrm{O}(p)$ and $\mathrm{Sn}(s)-\mathrm{O}(p)$ interactions would be enhanced and weakened, respectively. And both changes of intralayer effects would help open the band gap of $\beta-\mathrm{SnO}$ as illustrated in Fig. 6. In addition, the dehybridization of $\operatorname{Sn}(s)$ and $\operatorname{Sn}\left(p_{z}\right)$ orbitals would strengthen the stabilization effect of $\operatorname{Sn}\left(p_{z}\right)$ on the antibonding states of $\operatorname{Sn}(s)-\mathrm{O}(p)$ interaction shown in Fig. 6 and helps to open the band gap as well. Therefore, the oscillation of band gap in the pressure range of 9-13 GPa is the result of the competition of closing effect of interlayer $\mathrm{Sn}-\mathrm{Sn}$ interaction and the opening effect of intralayer Sn-O interaction. With the further increase of external pressure, the intralayer $\mathrm{Sn}-\mathrm{O}$ interaction became the dominant effect and opens the band gap continuously. This mechanism is evidenced by calculated wavefunction characters of CBM and VBM at different pressures (Fig. S13). The wavefunction characters of VB-WГ (VBM along the $\mathrm{W}-\Gamma)$ and $\mathrm{CB}-\mathrm{W} \Gamma(\mathrm{CBM}$ along the $\mathrm{W}-$ $\Gamma)$ at $0 \mathrm{GPa}$ reflect electron coupling between interlayer atoms, while the wave-function 
characters of VB- $Г \mathrm{Y}(\mathrm{VBM}$ along the $Г-\mathrm{Y})$ and $\mathrm{CB}-\Gamma \mathrm{Y}(\mathrm{CBM}$ along the $Г-\mathrm{Y})$ at 13 GPa trend to show the coupling of intralayer atoms.

\section{Conclusion}

Here in this work, we carried out a systematic theoretical study on the stability and electronic structure of SnO under external pressures. HSE06-TS-SCS and DFT-D3-BJ are respectively proved to be the best HSE- and PBE-based functionals for the relaxation of layered $\mathrm{SnO}$. A phase transition from $\alpha-\mathrm{SnO}$ to $\beta-\mathrm{SnO}$ is expected at around $80 \mathrm{GPa}$. Two high-pressure phases, P2/c- and Pnma-SnO, can be hopefully synthesized under high pressure and would transform to $\beta$-SnO after pressure releasing. Besides, the external pressure is demonstrated capable of modulating the band gap of $\beta$-SnO. A semiconductor-to-metal transition occurs in the low-pressure range of 0-9 GPa. In parallel with the pressure-induced variation of the band gap, the $\beta$-SnO is characterized by adjustable hole and electron mobilities. Moreover, $\beta$-SnO is expected to be a new transparent semiconductor at low pressure. Together these results provide important implication that high conductivity and transparency of $\beta-\mathrm{SnO}$ could be obtained upon applied external pressure (such as $9 \mathrm{GPa}$ ). Finally, the mechanism behind variation of the band gap was proposed as the competition of interlayer $\mathrm{Sn}-\mathrm{Sn}$ and intralayer Sn-O interactions. In summary, this work has been the one of the first attempts to thoroughly examine the performance of $\mathrm{vdW}$ functionals under high pressure, which will guide the study of layered structures under high pressure. Moreover, this work demonstrates the mightiness of high pressure on structural modulation and new allotrope discovery of $\mathrm{SnO}$, which would provide guidelines for the development of high effective materials in many structure-sensitive applications.

\section{Supporting Information}

Lattice parameters of $\mathrm{SnO}$ at the different pressure obtained in experiment, percentage changes of lattice parameters with respect to the experimental values at the different pressure, the phonon dispersions and band structures of $\mathrm{SnO}$ at the different pressure.

\section{Acknowledgment}


This work is supported by National Natural Science Foundation of China (Grants No. 51872242) and the Fundamental Research Funds for the Central Universities (No. D5000200142). V.A.B. thanks the Russian Science Foundation (Grant No. 16-1310158) for support of developing the network topological model. A.R.O. thanks the Russian Science Foundation (Grant No. 19-72-30043).

\section{Reference}

[1] Ogo Y, Hiramatsu H, Nomura K, et al. P-channel thin-film transistor using p-type oxide semiconductor, SnO. Applied Physics Letters 2008, 93: 032113.

[2] Idota Y, Kubota T, Matsufuji A, et al. Tin-based amorphous oxide: A high-capacity lithium-ionstorage material. Science 1997, 276: 1395-1397.

[3] Hosono H, Ogo Y, Yanagi H, et al. Bipolar conduction in $\mathrm{SnO}$ thin films. Electrochemical and Solid-State Letters 2011, 14: H13.

[4] Allen JP, Scanlon DO, Piper LFJ, et al. Understanding the defect chemistry of tin monoxide. Journal of Materials Chemistry C 2013, 1: 8194-8208.

[5] Peng H, Bikowski A, Zakutayev A, et al. Pathway to oxide photovoltaics via band-structure engineering of SnO. Applied Physics Letters Materials 2016, 4: 106103.

[6] Wang J, Umezawa N, Hosono H. Mixed valence tin oxides as novel van der Waals materials: Theoretical predictions and potential applications. Advanced Energy Materials 2016, 6: 1501190.

[7] Oganov AR, Glass C. Crystal structure prediction using ab initio evolutionary techniques: Principles and applications. The Journal of chemical physics 2006, 124: 244704.

[8] Oganov AR, Lyakhov AO, Valle M. How evolutionary crystal structure prediction works-and why. Accounts of Chemical Research 2011, 44: 227-237.

[9] Lyakhov AO, Oganov AR, Stokes HT, et al. New developments in evolutionary structure prediction algorithm USPEX. Computer Physics Communications 2013, 184: 1172-1182.

[10] Walsh A, Watson GW. Electronic structures of rocksalt, litharge, and herzenbergite SnO by density functional theory. Physical Review B 2004, 70: 235114.

[11] Zhang W, Oganov AR, Goncharov AF, et al. Unexpected stable stoichiometries of sodium chlorides. Science 2013, 342: 1502-1505.

[12] Zhang W, Oganov AR, Zhu Q, et al. Stability of numerous novel potassium chlorides at high pressure. Scientific Reports 2016, 6: 26265.

[13] Dong X, Oganov AR, Goncharov AF, et al. A stable compound of helium and sodium at high pressure. Nature Chemistry 2017, 9: 440-445.

[14] Giefers H, Porsch F, Wortmann G. Structural study of SnO at high pressure. Physica B: Condensed Matter 2006, 373: 76-81.

[15] Govaerts K, Saniz R, Partoens B, et al. Van der Waals bonding and the quasiparticle band structure of SnO from first principles. Physical Review B 2013, 87: 235210.

[16] Klimeš J, Bowler DR, Michaelides A. Chemical accuracy for the van der Waals density functional. Journal of Physics: Condensed Matter 2009, 22: 022201. 
[17] Grimme S, Antony J, Ehrlich S, et al. A consistent and accurate ab initio parametrization of density functional dispersion correction (DFT-D) for the 94 elements H-Pu. Journal of Chemical Physics 2010, 132: 154104.

[18] Alexandre T, Matthias S. Accurate molecular van der Waals interactions from ground-state electron density and free-atom reference data. Physical Review Letters 2009, 102: 073005.

[19] Alexandre T, Distasio RA, Roberto C, et al. Accurate and efficient method for many-body van der Waals interactions. Physical Review Letters 2012, 108: 236402.

[20] Alberto A, Reilly AM, Distasio RA, et al. Long-range correlation energy calculated from coupled atomic response functions. Journal of Chemical Physics 2014, 140: 150901.

[21] Peng H, Yang Z-H, Perdew JP, et al. Versatile van der Waals density functional based on a metageneralized gradient approximation. Physical Review X 2016, 6: 041005.

[22] Heyd J, Scuseria GE, Ernzerhof M. Erratum: "Hybrid functionals based on a screened Coulomb potential” [J. Chem. Phys. 118, 8207 (2003)]. The Journal of Chemical Physics 2006, 124: 219906.

[23] Grimme S, Ehrlich S, Goerigk L. Effect of the damping function in dispersion corrected density functional theory. Journal of Computational Chemistry 2011, 32: 1456-65.

[24] Kresse G, Furthmüller J. Efficient iterative schemes for ab initio total-energy calculations using a plane-wave basis set. Physical Review B 1996, 54: 11169-11186.

[25] Perdew JP, Burke K, Ernzerhof M. Generalized gradient approximation made simple. Physical Review Letters 1996, 77: 3865-3868.

[26] Blöchl PE. Projector augmented-wave method. Physical Review B 1994, 50: 17953-17979.

[27] Wang J, Hanzawa K, Hiramatsu H, et al. Exploration of stable strontium phosphide-based electrides: Theoretical structure prediction and experimental validation. Journal of the American Chemical Society 2017, 139: 15668-15680.

[28] Monkhorst HJ, Pack JD. Special points for Brillouin-zone integrations. Physical Review B 1976, 13: $5188-5192$

[29] Togo A, Oba F, Tanaka I. First-principles calculations of the ferroelastic transition between rutiletype and $\mathrm{CaCl}$-type $\mathrm{SiO} 2$ at high pressures. Physical Review B 2008, 78: 134106.

[30] Bučko T, Lebègue S, Gould T, et al. Many-body dispersion corrections for periodic systems: an efficient reciprocal space implementation. Journal of Physics: Condensed Matter 2016, 28: 045201

[31] Grimme S. Semiempirical GGA-type density functional constructed with a long-range dispersion correction. Journal of Computational Chemistry 2006, 27: 1787-99.

[32] Lyakhov AO, Oganov AR. Evolutionary search for superhard materials: Methodology and applications to forms of carbon and $\mathrm{TiO}_{2}$. Physical Review B 2011, 84: 092103.

[33] Yu S, Jia X, Frapper G, et al. Pressure-driven formation and stabilization of superconductive chromium hydrides. Scientific Reports 2015, 5: 17764.

[34] Blatov VA, Shevchenko AP, Proserpio DM. Applied topological analysis of crystal structures with the program package ToposPro. Crystal Growth \& Design 2014, 14: 3576-3586.

[35] Michael OK, Peskov MA, Ramsden SJ, et al. The Reticular Chemistry Structure Resource (RCSR) database of, and symbols for, crystal nets. Accounts of Chemical Research 2008, 41: 1782-1789.

[36] Blatov VA. Topological relations between three-dimensional periodic nets. I. Uninodal nets. Acta Crystallogr A 2007, 63: 329-43.

[37] Blatov VA, Golov AA, Yang C, et al. Network topological model of reconstructive solid-state transformations. Scientific Reports 2019, 9: 6007.

[38] Wang V, Xu N, Liu JC, et al. VASPKIT: A pre- and post-processing program for VASP code. 2019. 
[39] Saha S, Sinha TP, Mookerjee A. Electronic structure, chemical bonding, and optical properties of paraelectric $\mathrm{BaTiO}_{3}$. Physical Review B 2000, 62: 8828-8834.

[40] Sui X, Wang J, Duan W. Prediction of stoner-type magnetism in low-dimensional electrides. Journal of Physical Chemistry C 2019, 123: 5003-5009.

[41] Dion M, Rydberg H, Schroder E, et al. van der Waals density functional for general geometries. Phys Rev Lett 2004, 92: 246401.

[42] Klimeš J, Bowler DR, Michaelides A. Van der Waals density functionals applied to solids. Physical Review B 2011, 83.

[43] Koch E, Fischer W. Types of sphere packings for crystallographic point groups, rod groups and layer groups. Ztschrift Für Kristallographie 1978, 148: 107-152.

[44] Blatov VA, Proserpio DM. Topological relations between three-periodic nets. II. Binodal nets. Acta Crystallographica 2009, 65: 202-212.

[45] Tauc J. Optical properties and electronic structure of amorphous Ge and Si. 1969.

[46] Shao G, Jiang J, Jiang M, et al. Polymer-derived SiBCN ceramic pressure sensor with excellent sensing performance. Journal of Advanced Ceramics 2020, 9: 374-379.

[47] Arai T, Iimura S, Kim J, et al. Chemical design and example of transparent bipolar semiconductors. Journal of the American Chemical Society 2017, 139: 17175-17180.

[48] Wang F, Tan M, Li C, et al. Unusual pressure-induced electronic structure evolution in organometal halide perovskite predicted from first-principles. Organic Electronics 2019, 67: 89-94.

[49] Lu Y, Zhu S, Huang E, et al. Pressure-driven band gap engineering in ion-conducting semiconductor silver orthophosphate. Journal of Materials Chemistry A 2019, 7: 4451-4458 
Figures

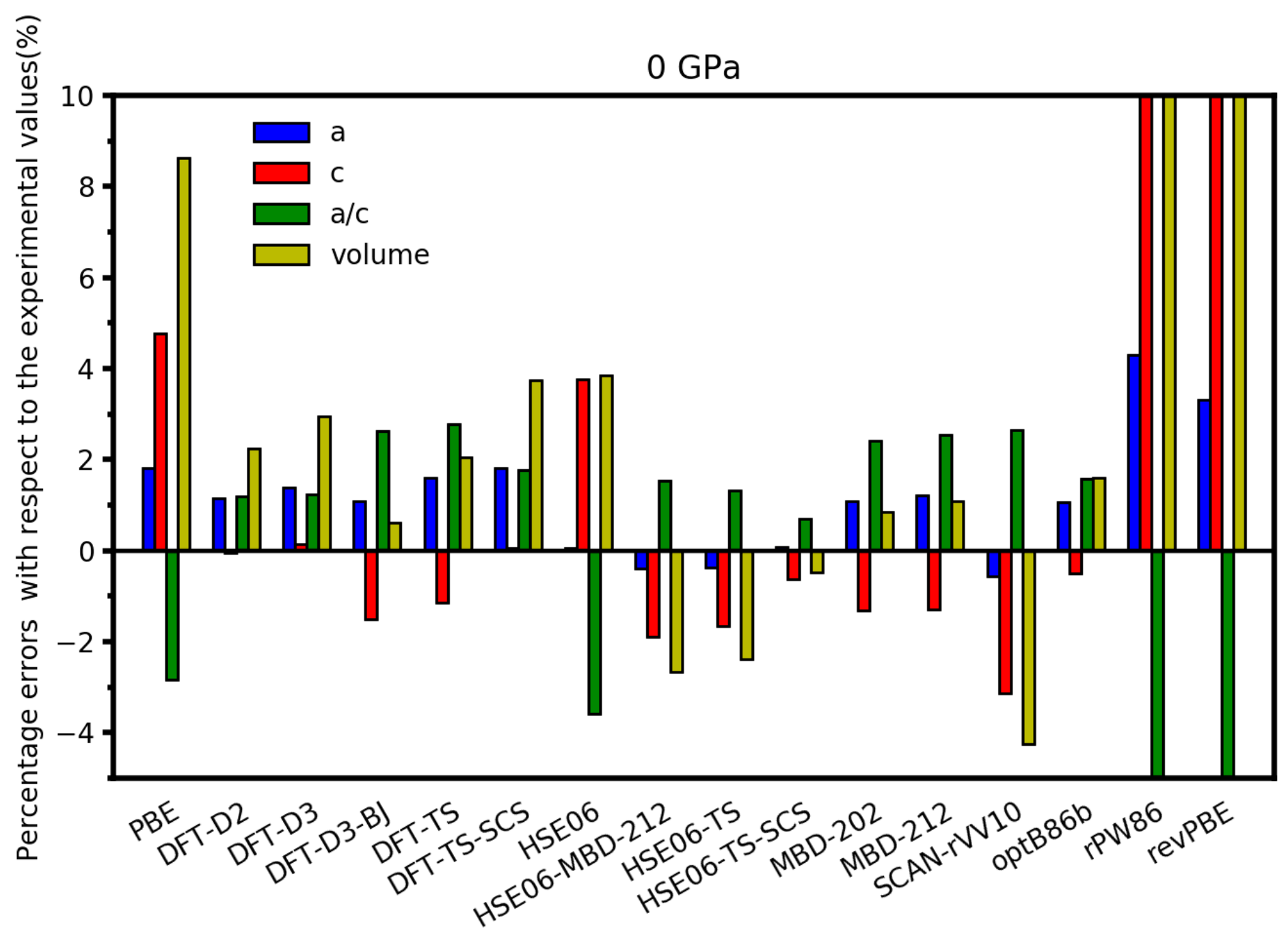

Figure 1

Percentage errors of calculated a and $\mathrm{c}, \mathrm{a} / \mathrm{c}$ ratio and volume of the unit cell of a-SnO using different functionals with respect to the experimental values at $0 \mathrm{GPa}$. 


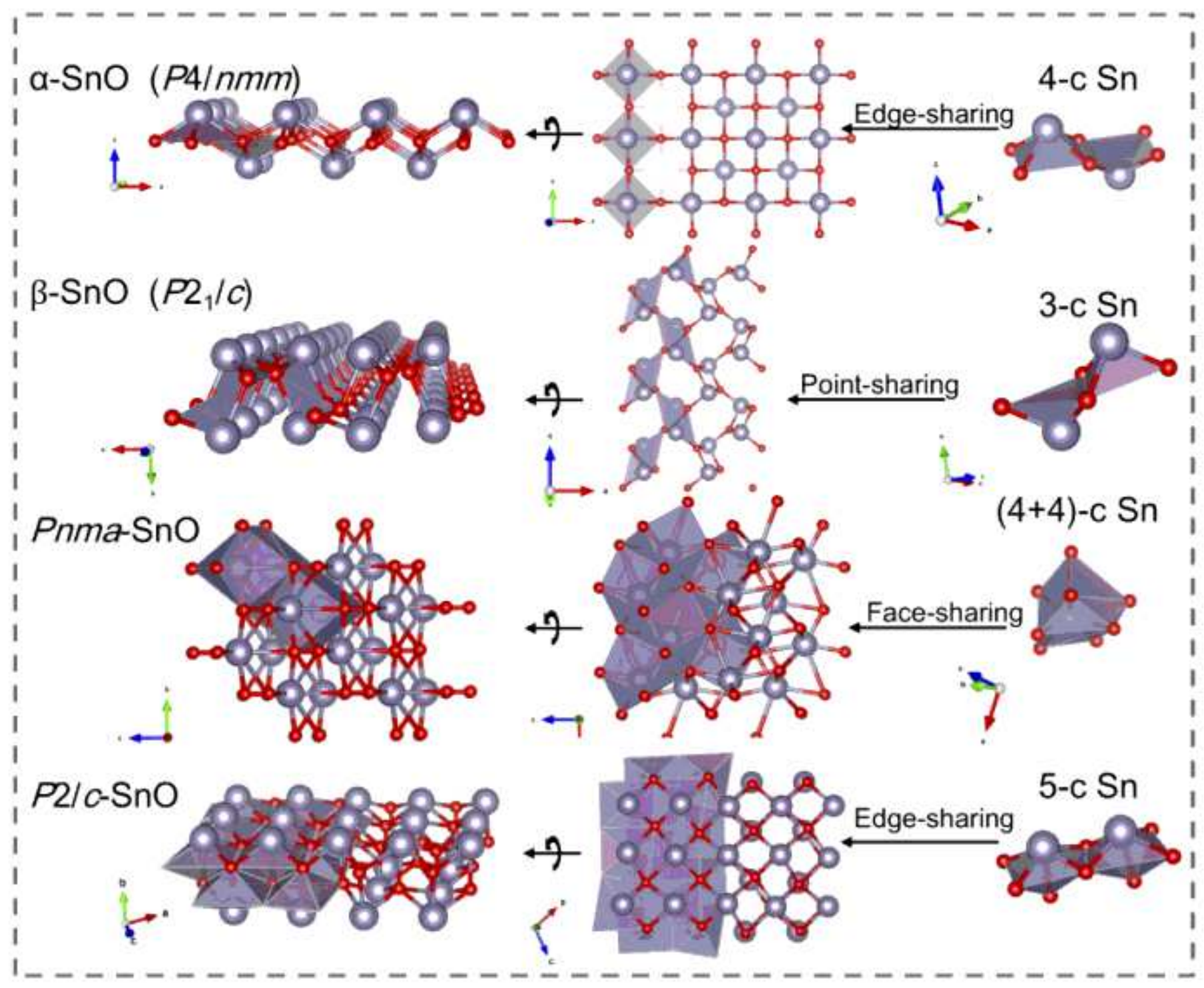

Figure 2

Crystal structures of a-SnO, $\beta-S n O$, Pnma-SnO and P2/c-SnO 


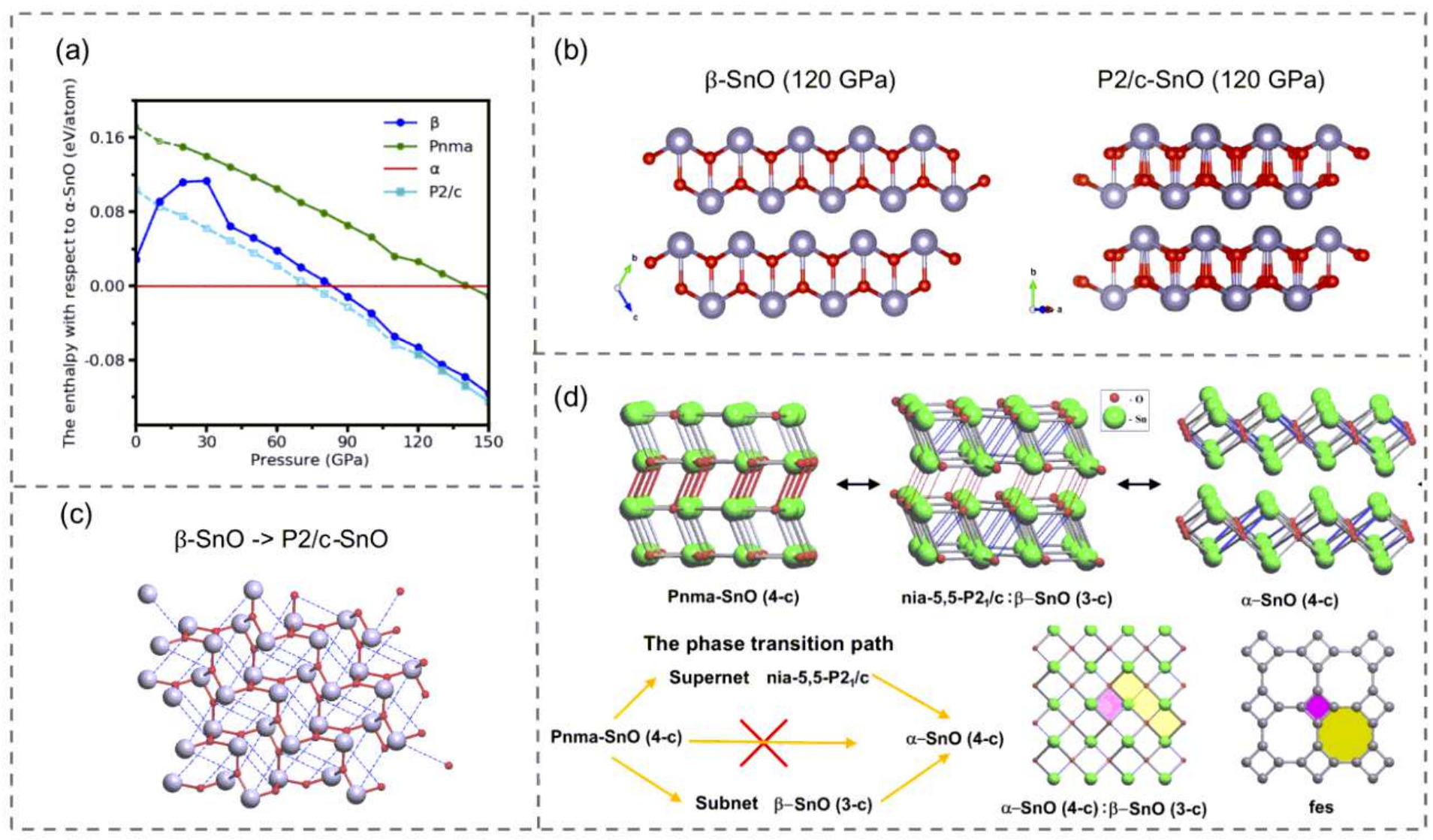

Figure 3

(a) The enthalpy per atom for predicted tin monoxides as a function of external pressure with respect to a-SnO. For Pnma- and P2/c-SnO, the dashed lines and hollow symbols indicate the dynamical instability at certain pressure. (b) A schematic diagram to demonstrate the structural similarity between $\mathrm{P} 2 / \mathrm{c}-\mathrm{SnO}$

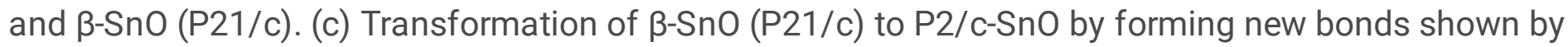
thin blue lines. (d) The phase transition from high-pressure Pnma-SnO to a-SnO through a supernet nia$5,5-\mathrm{P} 21 / \mathrm{c}$ or $\beta$-SnO. Breaking the blue or red bonds in the supernet (upper middle of $\mathrm{d}$ ) leads to $\mathrm{a}-\mathrm{SnO}$ or Pnma-SnO, respectively, while breaking both types of bonds gives rise to a-SnO; Relation between Pnma$\mathrm{SnO}$ and $\beta$-SnO layers: breaking thin red bonds in Pnma-SnO leads to the $\beta$-SnO fes topology (upper left of $d$ ). The idealized fes net consisting of four- and eight-membered rings highlighted by magenta and yellow (bottom right of $d$ ), respectively. These rings correspond to the rings of the same color in the bottom middle picture. 
(a)

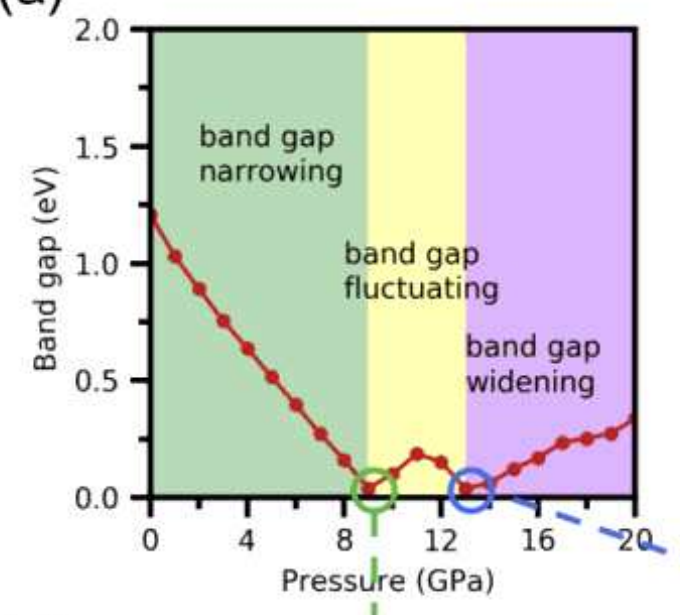

(c)

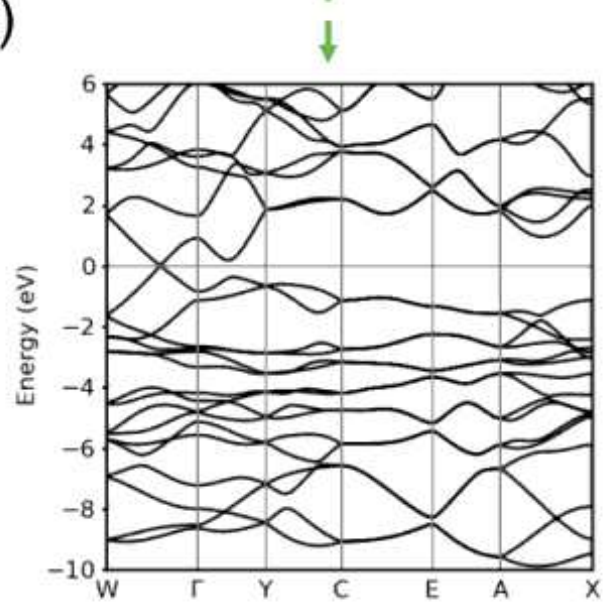

(e)

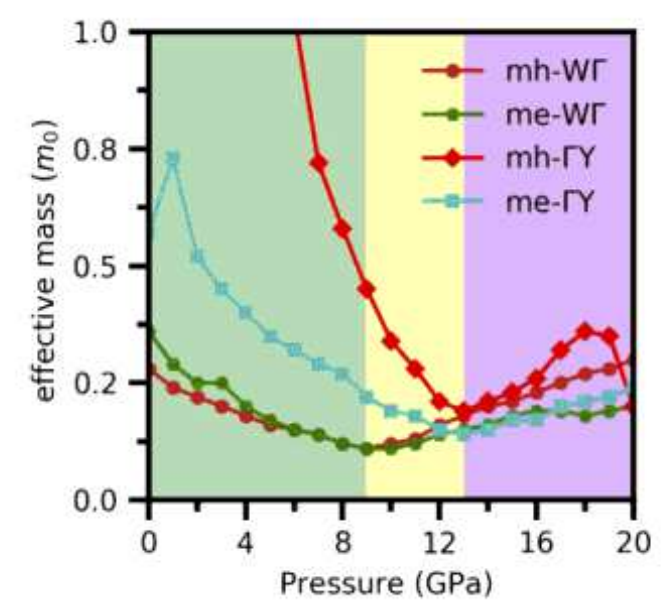

(b)
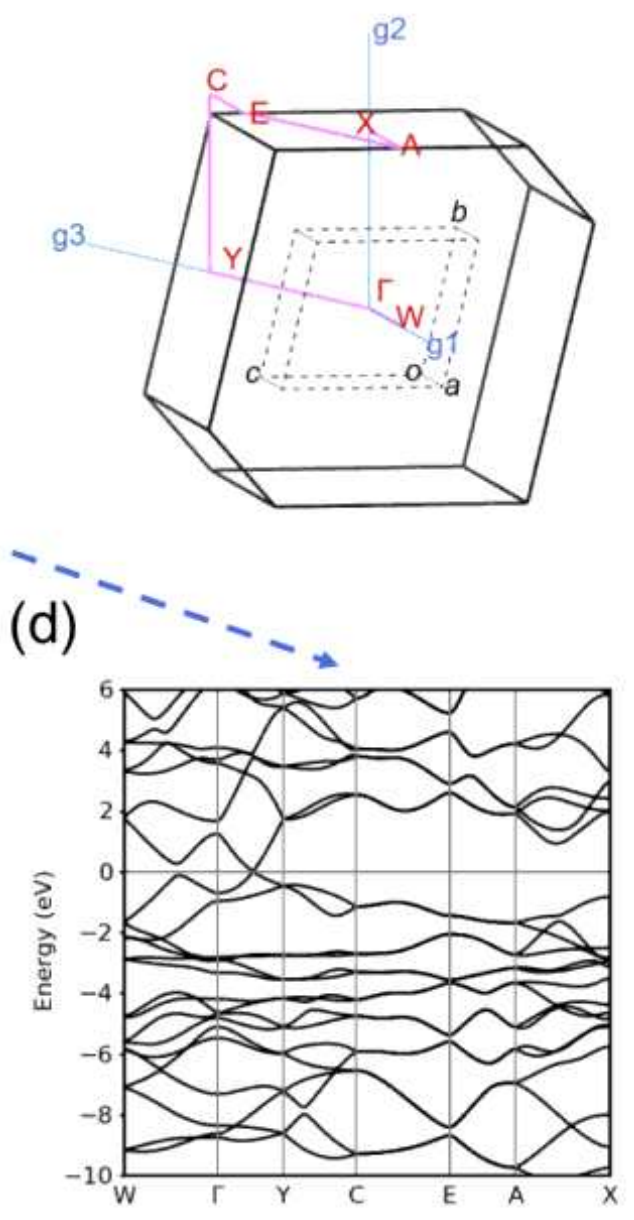

(f)

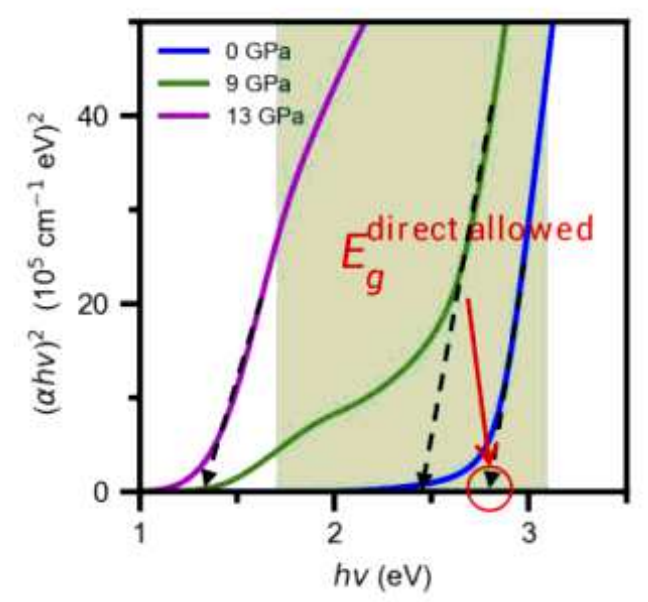

\section{Figure 4}

(a) The variation of calculated band gap of $\beta$-SnO using DFT-D3-BJ functional as a function of external pressure in the range of 0-20 GPa. (b) The Brillouin zone of $\beta$-SnO. The calculated band structures at pressures of (c) $9 \mathrm{GPa}$ and (d) $13 \mathrm{GPa}$. (e) Calculated effective mass of $\beta$-SnO along different highsymmetry directions; $\mathrm{mh}$ and me represent the effective mass of hole and electron, respectively. (f) Calculated absorption spectra, (ahv)2 vs hv, for $\beta$-SnO at different pressures, where $a$ and hv are the 
absorption coefficient and photon energy, respectively. The direct allowed band gap has been measured by extrapolating straight portion of the curves (ahv) 2 vs hv [45]. Notably, the edge of the absorption spectrum at different pressures were shifted $+0.69 \mathrm{eV}$ to fit the band gap estimated by the HSE06-TSSCS. The green area represents the energy range of visible light.

(a)

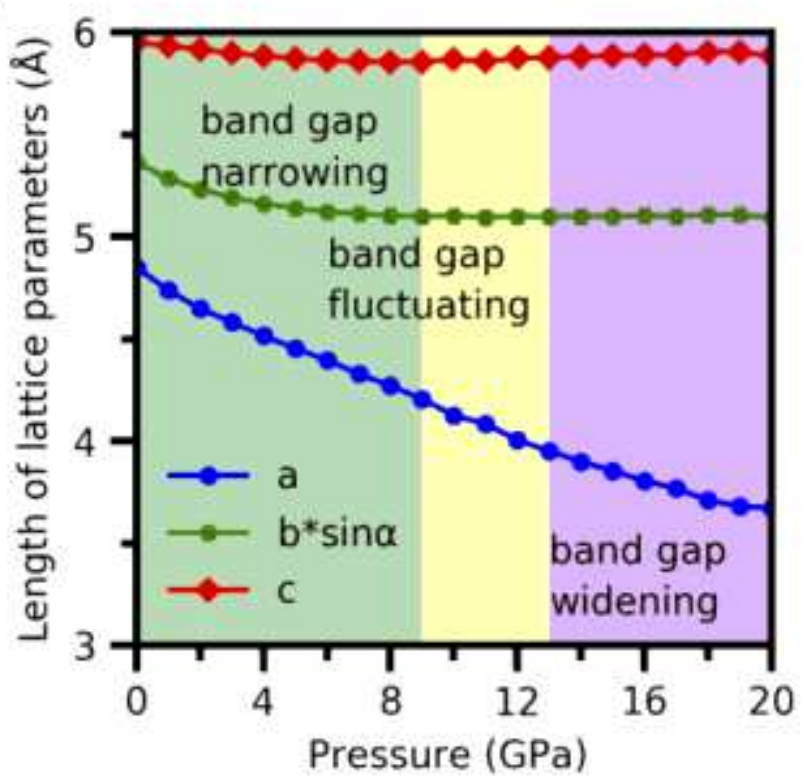

(c)

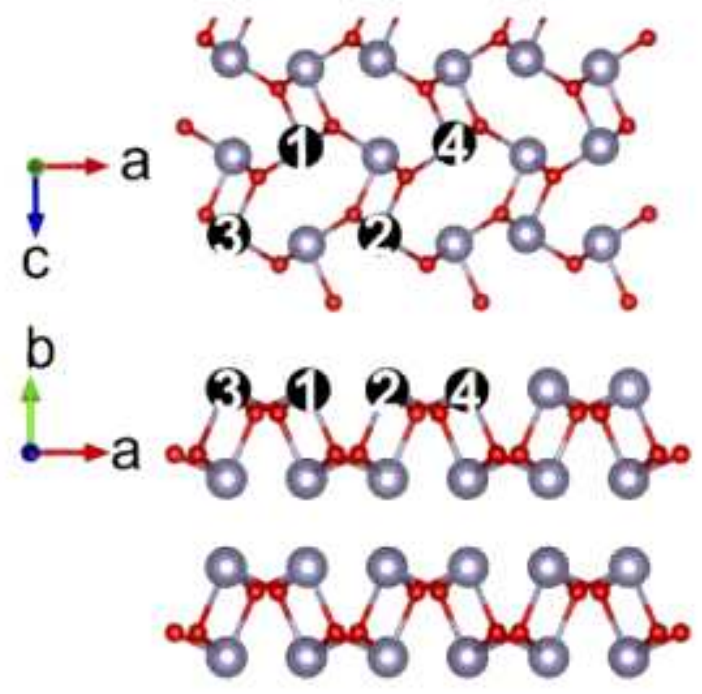

(b)

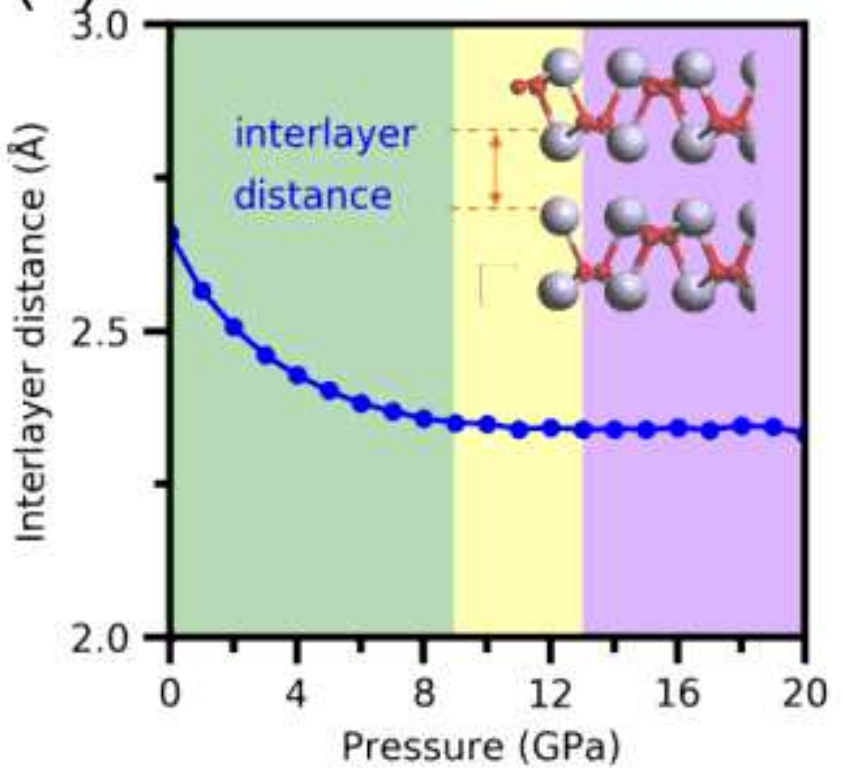

(d)

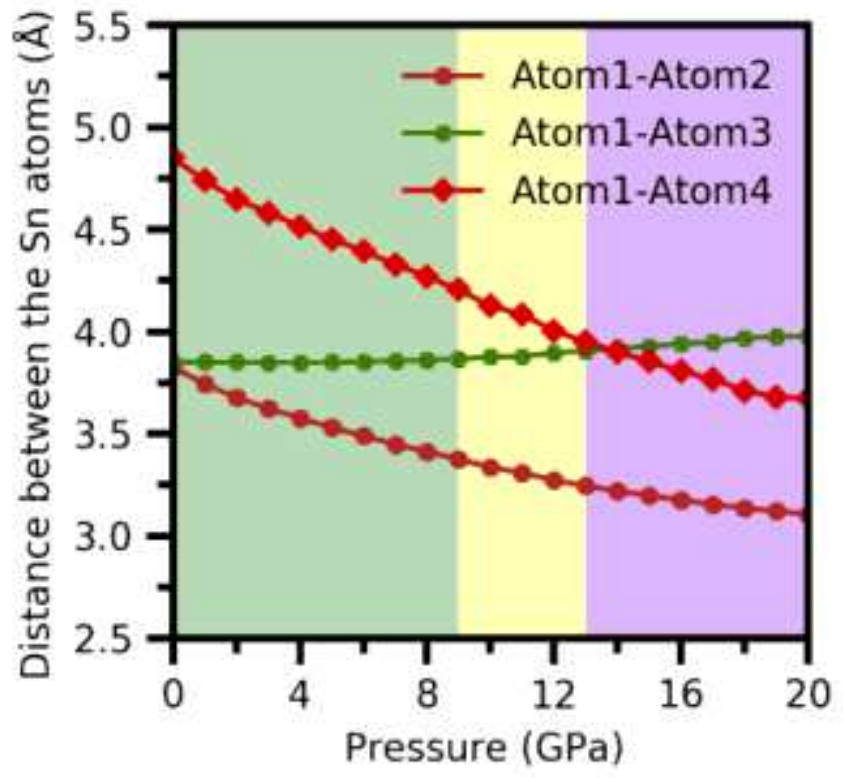

Figure 5

The variation of (a) lattice parameters, (b) interlayer distance and (c, d) distance between different Sn atoms on the same surface as a function of external pressure in the range of 0-20 GPa. 


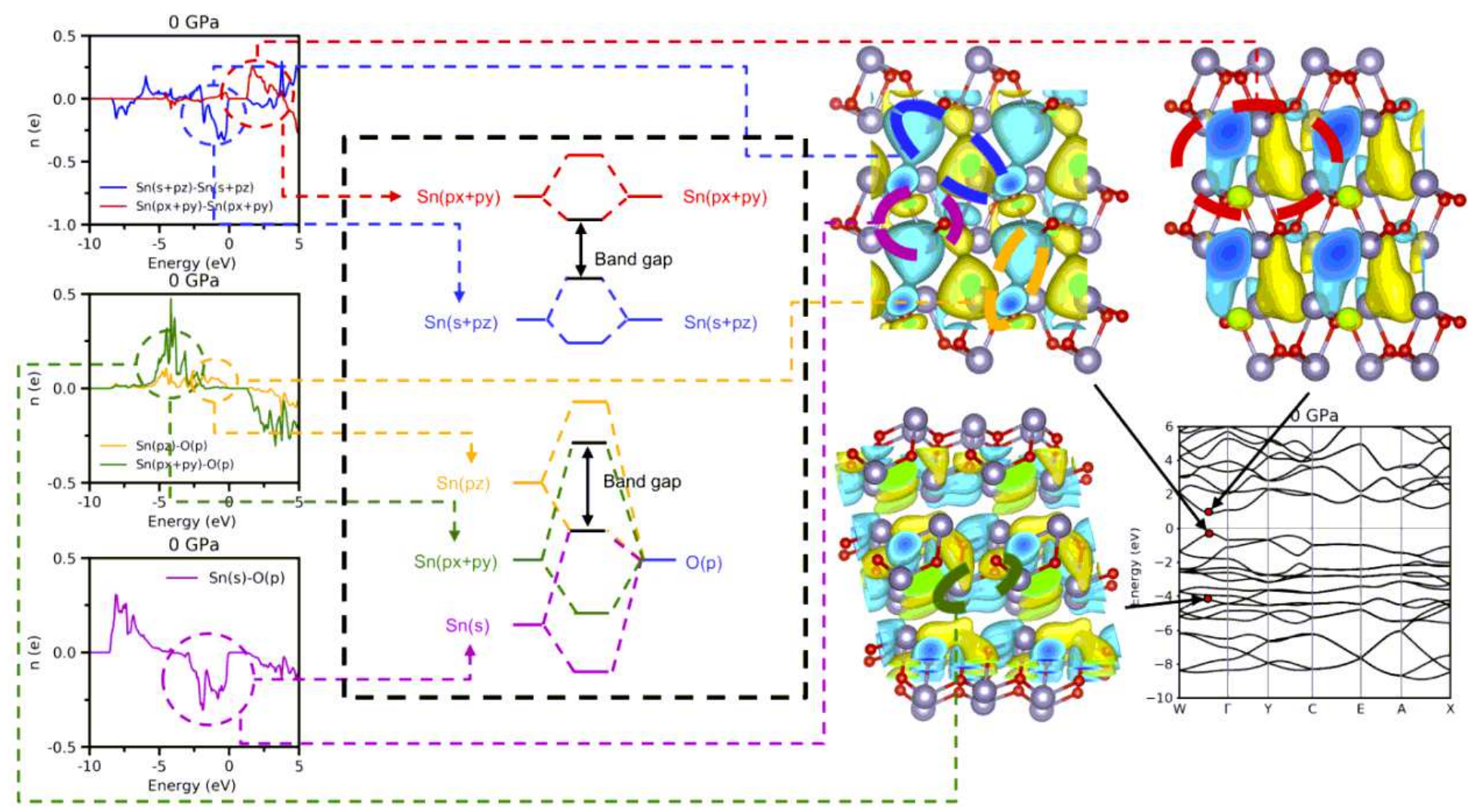

Figure 6

The calculated crystal orbital overlap populations (COOP), schematic diagram of orbital interactions and the wave functions at representative points of the band structure for $\beta-S n O$ at $0 \mathrm{GPa}$. In this work, axis $\mathrm{z}$ is perpendicular to the surface of SnO layer, while $\mathrm{x}$ and $\mathrm{y}$ are parallel to the axes a and c, respectively.

\section{Supplementary Files}

This is a list of supplementary files associated with this preprint. Click to download.

- Supplement2020009211.docx 